\title{
A Survey of Medium Access Mechanisms for Providing QoS in Ad-Hoc Networks
}

\author{
Marek Natkaniec, Senior Member, IEEE, Katarzyna Kosek-Szott, Member, IEEE, \\ Szymon Szott, Member, IEEE, and Giuseppe Bianchi
}

\begin{abstract}
In this survey we attempt to describe the Quality of Service (QoS) mechanisms employed by Medium Access Control (MAC) protocols designed for ad-hoc networks. We begin with background information: an overview of the related work, the definition of QoS and QoS-related metrics, a general description of contention-free and contention-based protocols for wireless networks, a discussion of issues affecting QoS provisioning in ad-hoc networks, as well as a novel classification of the QoS mechanisms. Then, each mechanism is briefly explained and implementation examples from different protocols are provided. Furthermore, a separate section is devoted to the completed and ongoing standardization work in the field. Afterwards, an extensive comparison of salient features, advantages and disadvantages of all described MAC mechanisms is given in order to guide future protocol designers. Finally, we comment on the most probable future research directions. Based on the presented survey, we observe that QoS provisioning is not only challenging but also a significant contemporary research problem. The protocol designs presented in the literature usually involve trade-offs between certain metrics, and currently there is no ideal solution which deals with all the issues affecting ad-hoc networks. Therefore, we trust that this survey will be of great help to designers of future QoS-aware protocols.
\end{abstract}

Index Terms-Ad-hoc networks, decentralized wireless networks, Quality of Service, MAC protocols, QoS mechanisms, QoS challenges, survey, comparison study, classification

\section{INTRODUCTION}

A D-HOC networks ${ }^{1}$ have become increasingly popular in recent years. This is related to their easy deployment and fast configuration. They can usually be set-up in environments where the deployment of a planned network is difficult or not economically feasible (e.g., disaster areas, training grounds, schools, conference sites, hotels, airports). An ad-hoc network is composed of wireless, potentially mobile stations, which do not require the use of any wired infrastructure or centralized administration. The initialization and administration of the

Manuscript received 10 January 2012; revised 8 May 2012

M. Natkaniec, K. Kosek-Szott, and S. Szott are with the Department of Telecommunications, Faculty of Electrical Engineering, Automatics, Computer Science and Electronics, AGH University of Science and Technology, al. Mickiewicza 30, 30-059 Krakow, Poland (e-mail: \{natkanie, kosek, szott\}@kt.agh.edu.pl).

G. Bianchi is with CNIT / University of Rome "Tor Vergata", Department of Electronics Engineering, Via del Politecnico, 1, 00133 Roma, Italy (e-mail: giuseppe.bianchi@uniroma2.it).

Digital Object Identifier 10.1109/SURV.2012.060912.00004

${ }^{1}$ Throughout this paper we use the term ad-hoc networks to denote decentralized Wireless Local Area Networks (WLANs). However, it should be noted that this term may also refer to slightly different network types, e.g., Wireless Sensor Networks (WSN), Wireless Personal Area Networks (WPAN), and Wireless Body Area Networks (WBAN). This paper focuses on WLAN technologies, therefore, other networks are out of its scope. network is performed in a distributed, peer-to-peer fashion. Stations are free to move randomly and organize themselves arbitrarily. Therefore, the network's topology may change rapidly and unpredictably. As a result, the ad-hoc network should be able to quickly adapt to a varying number of stations. Additionally, because of limited transmission range, wireless ad-hoc networks are usually non-fully connected. Therefore, data may need to travel through several intermediate stations in a multi-hop fashion before it reaches its destination. Ad-hoc networks were initially proposed for military applications, however, they have become of commercial interest to telecommunications companies, especially in scenarios in which they are integrated through gateways to the wired infrastructure [1].

The key requirement for ad-hoc networks to become even more useful and popular is to support applications which need QoS. With an increasing demand for multimedia applications it is expected that ad-hoc networks will provide correct traffic differentiation and support for heterogeneous services. Multimedia traffic is usually delay-sensitive and audio-visual content requires the end-to-end delay to be below a certain limit. Each real-time frame that belongs to a multimedia flow has to reach the final destination within a specified deadline, after which it becomes useless. Therefore, end-to-end QoS assurances can only be provided if each station in the network provides the means for offering QoS guarantees. Although much progress has been done in QoS for wireless networks in general, there are still many unsolved problems that appear in ad-hoc networks. Some of these challenges include limited transmission range, lack of centralized control, hidden, exposed and intruder terminal problems, battery and computational power constraints, frame losses due to collisions and transmission errors, multi-hop operation, unidirectional links and synchronization issues. Therefore, a QoS-aware wireless ad-hoc network requires a precisely designed Medium Access Control (MAC) protocol.

The medium access protocol is one of the most important aspects of any ad-hoc network because it has a direct bearing on how efficiently and reliably data can be transmitted. The MAC protocol should address the contention and collision problems among mobile stations and at the same time effectively utilize the communication channel. Developing a QoS-aware MAC protocol is not a trivial task because a good balance should be assured between protocol complexity, method of QoS reservation, available traffic classes, signaling overhead, supported QoS metrics, fairness, efficient use of resources, and consumed energy. 
We therefore present a comprehensive survey of the medium access mechanisms recently proposed in the literature that allow for QoS provisioning in ad-hoc networks. To narrow the scope of this survey, we have focused only on those mechanisms which support multiple traffic categories. More than seventy protocols have been surveyed to explain the operation of the various medium access mechanisms. Other aspects related to MAC protocols (such as fairness, powersaving, security) are out of the scope of this survey.

The remainder of this paper is structured as follows. Section II provides an overview of existing surveys of MAC protocols for ad-hoc networks. In Section III we discuss different QoS approaches, and commonly employed MAC layer QoS metrics. This is followed by a classification of QoS-aware MAC protocols, an explanation of the basic contention and contention-free access protocols, and description of issues that affect QoS provisioning. Section IV describes the mechanisms which may be employed in MAC protocols to provide QoS in ad-hoc networks. All currently standardized MAC protocols with QoS support are briefly discussed in Section V. We directly compare different protocol features in tables as showed in Section VI. Section VII includes interesting figures and analysis conducted from that comparison. Then, open issues and future research directions are identified in Section VIII. Finally, Section IX concludes the whole paper.

\section{RELATED WORK}

There already exist several surveys of MAC protocols for ad-hoc networks available in the literature [2], [3], [4], [5], [6], [7], [8], [9], [10], [11]. However, only a few of them mention QoS issues, and none of them concentrates exclusively on medium access mechanisms for supporting QoS at the MAC layer.

An early survey presented by Perkins and Hughes in [6] contains a description of network mechanisms and protocols (i.e., signaling and resource reservation, QoS routing, and MAC) that should closely cooperate to provide QoS in adhoc networks. Unfortunately, only a few QoS-aware MAC protocols are shortly mentioned in the paper. The survey presented by Jawhar and $\mathrm{Wu}$ in [7] considers the problem of QoS support in Time Division Multiple Access (TDMA) based ad-hoc networks. Different layers of the networking model are analyzed and categorized with the main emphasis put on the network layer and QoS routing algorithms. Reddy et al. [8] analyzed several solutions proposed in the literature for QoS provisioning in ad-hoc networks. The existing QoS approaches are classified according to several criteria such as interaction between routing protocol and resource reservation signaling, interaction between network and MAC layer, and updating routing information. A significant part of the survey contains a description of QoS frameworks and routing protocols and only several MAC protocols are presented. A fairly comprehensive overview of MAC protocols for ad-hoc wireless networks is provided by Kumar, Raghavan, and Deng in [9]. They analyzed a large number of non-QoS and QoSaware protocols and looked into issues of collision resolution, power conservation, multiple channels, and advantages of using directional antennas. The characteristics and operating principles of MAC protocols were considered. About ten QoSaware MAC protocols were very shortly explained and several more were presented in greater detail. Unfortunately, due to large number of MAC protocols reviewed in the paper, their comparison is very limited and qualitative performance is showed only for some of the protocols. In [10], Tsigkas and Pavlidou studied the limitations and merits of mechanisms that have been proposed towards embedding QoS support in distributed wireless MAC protocols. Four medium access protocols (Priority Broadcast for DCF, EY-NPMA, EDCA, and Adaptive $m$-ary Tree algorithms with Priority Broadcast) were analyzed and compared using simulation experiments. The survey presented in Abbas and Kure [11] also examined different issues and challenges involved in providing QoS in ad-hoc networks. They discussed methods of QoS provisioning taking into consideration MAC, network, and cross layer solutions. A variety of protocols for admission control and scheduling proposed in the literature were also reviewed. About ten MAC protocols with different features belonging to one of four groups, namely Carrier Sense Multiple Access with Collision Avoidance (CSMA/CA), IEEE 802.11, TDMA, and Code Division Multiple Access (CDMA) were shortly described and compared. An overview of existing QoS solutions for multihop ad-hoc networks is presented by Natkaniec, Kosek-Szott, and Szott in [12]. The authors analyzed QoS aspects at three layers: physical, data link, and network. Additionally, crosslayer solutions were discussed. Unfortunately, only a few QoSaware MAC protocols were studied.

To the best of our knowledge, this is the first survey on medium access mechanisms that support QoS provisioning in ad-hoc networks. Moreover, we present these mechanisms and their protocol-specific implementation instead of describing each protocol individually. We have also identified that only few papers include an overview of some QoS-aware protocols, without any comparison between them. Moreover, we are convinced that a lot of research has been carried out in this area since the mentioned surveys appeared and a number of new protocols, issues, and solutions have emerged.

\section{BACKGROUND}

The fundamental service model of the Internet was based on the best effort delivery of packets. The reliable delivery of data was considered as the most important goal of the first Internet architecture. This architecture was also based on the concept that flow control will be performed in case of congestion at any part of the network. In a best effort network all users obtain an unspecified variable bit rate and delivery time, which depend on the traffic load in the network. This also means that the network does not guarantee that the service will be provided or that a user will obtain a guaranteed QoS level. Best effort delivery was completely sufficient for the first data applications, however, multimedia applications (such as VoIP, videoconferencing, video streaming, interactive gaming) require specific throughput, very high reliability in terms of limited packet loss, and/or bounded jitter and delay. Unfortunately, the best effort service cannot support such sophisticated requirements. Therefore, new mechanisms have to be developed to support the desired QoS levels for emerging 
modern applications. This is an especially demanding challenge for wireless networks characterized by unpredictable channels, unreliable links and extremely difficult for ad-hoc networks, where some additional unique challenges exist, such as station mobility, scalability, limited bandwidth, power and computational constraints, hidden and exposed stations.

\section{A. QoS provisioning}

Traditionally, QoS revolves around two different models: one aimed at providing strict per-flow guarantees at the expense of supplementary implementation and deployment complexity, and a second one, simpler but more loose, devised to differentiate the delivery of aggregate traffic classes. Indeed, these two models have been both considered for the Internet, in a large standardization effort carried out about 15 years ago by the Internet Engineering Task Force (IETF): integrated services architecture (IntServ) [13] and differentiated services (DiffServ) [14].

IntServ supports a set of specific algorithms and scheduling techniques which allow for strict QoS guarantees. In this model, the Resource Reservation Protocol (RSVP) is employed to request and reserve resources through a network. IntServ requires deterministic capacities assigned to each flow. If QoS is provided to flows individually, it is called individual QoS. It is also possible to provide QoS for a number of flows taken together. This is called aggregate QoS.

In Diffserv, prioritization is realized on a per-frame basis. DiffServ can be easily used to provide low-latency to critical network traffic such as voice or video streaming while providing simple best-effort service to non-critical traffic such as file transfers or web browsing. DiffServ, in contrast to IntServ, is a class-based mechanism for traffic management which operates on the principle of traffic classification, where each data frame is located into a specific number of traffic classes. DiffServ uses a mechanism that classifies and marks frames which belong to a specific class. Every intermediate station in the network that supports DiffServ implements Per-Hop Behaviors (PHBs), which define the frame forwarding properties associated with a class of traffic. Different forwarding properties can assure transmission of low-loss, low-latency, or any other traffic type. The most important advantage of DiffServ is that it is easy to setup and it does not require reservations for each traffic flow. There are also some drawbacks of DiffServ such as difficult prediction of the end-to-end behavior, dropping frames in case of congestion, and costly memory requirements since every station should behave as a source and an intermediate station.

Two principal approaches to assure QoS in wireless networks can be also defined at the MAC layer: prioritized, based on DiffServ and parameterized, based on IntServ. QoSaware MAC protocols for ad-hoc networks mostly utilize the prioritized approach due to the distributed nature of ad-hoc networks.

In this approach, violations of QoS provisioning are allowed over limited periods of time, however, the session QoS requirements are held over the total transmission time. This means that the value of QoS parameters may vary during the transfer of data frames by the MAC data service. There are a number of applications (e.g., equipped with internal buffers) that can be successfully used with such short time QoS violations. QoS assurance in this approach can also be strengthened using dedicated scheduling algorithms. Unfortunately, scheduling algorithms designed for wired networks cannot be directly used in ad-hoc networks since there is no central management point where all the required data (e.g., number of active stations and sessions, types of traffic, queue usage) can be collected to perform scheduling decisions. Therefore, a QoS-aware MAC protocol designed for ad-hoc networks should closely cooperate with the scheduling algorithm. The information obtained by the MAC protocol about other stations transmitting in the neighborhood allows for appropriate scheduling and improved prioritized QoS assurance. To summarize, the prioritized QoS approach should be considered in ad-hoc networks as the default service differentiation method based on a reservationless approach.

In the parameterized approach, QoS requirements are strict and can be expressed in terms of quantitative values, such as data rate, jitter, and delay bounds. These values are expected to be met by the MAC data service during the transfer of data frames between stations. Parameterized QoS utilizes the concept of flows to control bandwidth sharing among different traffic classes and provide deterministic QoS guarantees. Users can specify the QoS requirements of their applications by setting appropriate system QoS parameters. Network stations should fulfill these requirements for each flow. Therefore, parameterized QoS requires deterministic capacities to be assigned to each flow. This is to ensure that already admitted flows remain unaffected. There are four mechanisms defined within this approach to provide deterministic QoS guarantees: classification, scheduling, admission control, and reservation setup. Unfortunately, parameterized QoS provisioning encounters problems with the inherent lack of strict QoS guarantees in distributed MAC protocols, where the transmission is usually stochastic, unpredictable and prone to collisions.

\section{B. QoS and performance metrics}

The level of QoS provisioning is usually based on parameters or constraints, often known as QoS metrics. These QoS metrics may be defined separately for the different layers of the OSI model. Application layer QoS metrics show the QoS requirements of the user application. Network layer QoS metrics represent the quality of the end-to-end path. Finally, MAC layer QoS metrics indicate the quality of the link in the network. There is a strong relation between all theses metrics, e.g. the QoS metrics of an end-to-end path highly depend on the QoS metrics of links on the selected path. The most common QoS metrics defined at the MAC layer that should be considered while evaluating QoS-aware MAC layer mechanisms include: minimum throughput, maximum frame delay, maximum variation of frame delay (jitter), and maximum frame loss ratio. Their definitions are as follows:

- Minimum throughput (measured in bits/s): data throughput required at the MAC layer to assure correct operation of an application. This metric is highly related to the volume of information a single station can transmit over the wireless channel per unit of time. 
- Maximum frame delay (measured in seconds): the period of time a frame waits at the MAC layer until its successful transmission. This waiting period includes the MAC queuing delay (together with contention delay), transmission delay (together with propagation and processing delays) and possible MAC layer retransmission delay (if a frame is lost or corrupted, then no valid acknowledgement is received and the overall delay increases).

- Maximum variation of frame delay (known as jitter) (measured in seconds): defined as the difference between the upper bound of frame delay (including MAC queuing, transmission and retransmission delays) and the minimum delay, which is determined by the frame transmission delay (including propagation delay).

- Maximum frame loss ratio (measured in percents): the maximum tolerable fraction of frames that can be lost at the MAC layer. Frame losses can result from the excess of the frame retransmission limit (usually defined by the MAC protocol) during periods of inadequate channel quality or in case of collisions caused by a large number of contending stations, MAC queue overflow when congestion occurs, or timeouts of real-time frames.

Other performance goals that influence QoS and can be fulfilled at the MAC layer include: minimizing collisions, maximizing parallel transmissions, maximizing link stability, minimizing energy consumption, and maximizing transmission reliability. It should be noticed that an application may request a particular QoS by specifying its requirements in terms of one or more of the above QoS metrics. A proper routing protocol and admission control mechanism, which are out of the scope of this paper, should admit such a request only if a station (or network for multi-hop transmission) can fulfill these requirements.

\section{Issues Affecting QoS in Wireless Ad-hoc Networks}

Despite their unique advantages, wireless ad-hoc networks have multiple characteristics which pose problems for providing QoS. These characteristics are directly responsible for the multitude of QoS MAC protocols which have been proposed in the literature. The most important of these issues are described below. They are categorized based on their cause. Additionally, the impact on the design of QoS MAC protocols is given.

- Inherent issues

- Unreliable wireless channel - poor radio channel quality is related to interference, thermal noise, shadowing, multi-path fading, the near-far problem, and the capture effect. All these issues lead to retransmissions and varying transmission rates. Admission control and providing strict QoS guarantees are therefore challenging.

- Application requirements - applications have various requirements of which a QoS MAC protocol should be aware in order to fulfill them.

- Multiple traffic types - there are many types of traffic in a network, each having various frame characteristics (e.g., length, generation distribution). QoS MAC protocols may adopt a different channel access approach for various traffic types (Section IV-F1).

- Technology-based issues

- Half-duplex radio transceivers - with current radio technology, collisions cannot be detected directly but only after a certain timeout. Therefore, various confirmation schemes can be applied, including refraining from any acknowledgement for real-time data in order to increase channel efficiency.

- Multiple channels - stations equipped with more than one wireless interface require more complex MAC protocols but can provide higher network capacities.

- Directional antennas - stations equipped with directional antennas can have increased transceiver performance and reduced interference. However, this may simultaneously create hidden stations and thus have a detrimental impact on QoS.

- Deployment-based issues

- Multi-hop topology - due to the limited range of wireless technologies, a multi-hop approach is necessary to ensure full connectivity. This results in increased collisions (caused by hidden stations), inefficient bandwidth utilization (caused by exposed stations), and contention between locally-generated and forwarded traffic. These problems are so critical that they are often explicitly addressed by QoS MAC protocols.

- Mobility - station movement can result in collisions (despite prior channel reservation), link breakage, variation in the number of contending stations, and the need for handover mechanisms. Traffic control mechanisms can be defined to alleviate these problems.

- Limited energy supply - mobile stations often use batteries. MAC protocols should, therefore, employ power saving (i.e., a sleep mode), avoid transmissions which result in collisions, and keep transmission power levels at minimum. However, all this has to be addressed without loss of QoS support.

- Limited computational power - mobile devices are usually limited in computational power. Therefore, QoS MAC protocols cannot use highly complex algorithms.

- Network size - the increase of the number of stations generates significant overhead related to the discovery and maintenance of neighboring links. This may have an impact on end-to-end delay.

- Medium access-based issues

- Lack of centralized coordination in accessing a shared channel - stations use dynamically set up point-to-multipoint links which are not governed by a central authority. This means that QoS MAC protocols need to operate in a distributed manner often relying only on locally available information.

- Fairness - the medium access protocol needs to make sure that no stations are favored over others (e.g., that none have seized the channel) and that 
traffic of the same priority has the same transmission probability.

- Synchronization issues - QoS protocols may require accurate timing. Since ad-hoc networks lack a centralized coordinator, each station must be able to separately perform the synchronization, e.g., through GPS.

- Power control - stations may be able to dynamically change their transmission power. Station connectivity increases with transmission power, however, interference does as well. Therefore, QoS MAC protocols may seek to optimize certain values (e.g., the minimum acceptable transmission power).

- Signaling - performing reliable yet efficient (i.e., with minimum overhead) MAC layer signaling is a key aspect of all QoS MAC protocols.

- Misbehavior - selfish stations may not adhere to the MAC protocol, which can impact QoS provisioning.

\section{Distributed Coordination Function}

A number of QoS-aware MAC mechanisms (described later in this paper) are extensions of the Distributed Coordination Function (DCF). DCF was the first contention-based channel access protocol standardized for IEEE 802.11 ad-hoc networks. The basic mechanisms of DCF are explained in details next.

DCF is a distributed protocol, i.e., each station contends for access to the wireless channel, and enables asynchronous data transfer without QoS guarantees. It utilizes Carrier Sense Multiple Access with Collision Avoidance (CSMA/CA) [15]. Before each transmission, stations check the status of the wireless channel. If the channel is idle for a predefined amount of time, the station is allowed to transmit. However, transmitted frames may collide. Additionally, because stations cannot sense the channel while transmitting data, detection of colliding frames is more complicated than in wired CSMA/CD-based networks (e.g., Ethernet). Therefore, in DCF after each successful data transmission an acknowledgement frame (ACK) is sent by the destination. If the ACK frame is not received in a predefined time, a retransmission of the DATA frame is scheduled.

DCF can optionally employ the Request to Send/Clear to Send (RTS/CTS) frame exchange. Before each transmission of DATA the source station transmits an RTS control frame and the destination station transmits a CTS control frame in response to that RTS. Both RTS and CTS frames contain a Duration field, which defines the reservation time of the wireless channel required to transmit a DATA frame and an ACK frame. Stations overhearing the RTS and CTS frames learn of the channel reservation time and set their Network Allocation Vectors (NAVs) accordingly. After the reception of the CTS frame, the source station starts the transmission of its DATA frame, which is acknowledged by an ACK frame. The described procedure is called the four-way handshake mechanism and was proposed in the IEEE 802.11 standard to minimize the number of collisions caused by hidden stations [16]. The QoS-aware MAC protocols frequently implement this mechanism, e.g., in order to reserve bandwidth or a transmission period, negotiate the data channel, transmit additional
QoS-related information (e.g., transmission power, additional acceptable noise).

IEEE 802.11 defines four time intervals (called InterFrame Spaces, IFSs) in order to provide priority levels for access to the wireless channel. They are the following:

- Short InterFrame Space (SIFS) - the shortest IFS, used prior to transmissions of ACK frames and Clear To Send (CTS) frames, as well as before a response to polling by the Point Coordination Function (PCF). PCF is explained in Section III-E.

- Point Coordination Function InterFrame Space (PIFS) used to give priority to PCF channel access over DCF channel access.

- DCF InterFrame Space (DIFS) - used by stations operating under DCF to transmit DATA and RTS frames.

- Extended InterFrame Space (EIFS) - used after erroneous transmissions of frames.

In order to minimize the probability of collisions during contention between multiple wireless stations, DCF combines CSMA/CA with a random backoff procedure. The general description of DCF operation is as follows. Each time when a station intends to transmit a frame it must first determine if the channel is idle or busy. If the channel is busy the station has to defer until it is idle for DIFS. After this happens, the station chooses a random BackoffTime for an additional deferral before transmission. The BackoffTime is a random number of timeslots chosen in the following way:

$$
\text { BackoffTime }=\operatorname{rand}[0 ; C W] \times \text { SlotTime, }
$$

where $\mathrm{CW}$ is the Contention Window parameter and SlotTime is the length of a single timeslot. During the backoff period the station determines whether the channel is busy or idle. Until the channel is idle, the backoff value decreases by one after each time slot. Otherwise the procedure is suspended. After the backoff counter reaches zero, the station is allowed to transmit.

In case of DCF, before and during the backoff procedure, the channel has to be idle for at least a DIFS time period (if the previously detected transmission was correct) or an EIFS time period (if the previously detected transmission failed). Additionally, the initial value of $\mathrm{CW}$ is set to $C W_{\min }$. After each unsuccessful transmission attempt, the $\mathrm{CW}$ value is increased exponentially until it reaches $C W_{\max }$. After each successful transmission the value of $\mathrm{CW}$ is reset to $C W_{\min }$. The $\mathrm{DCF}$ BackoffTime is calculated using the following formula:

$$
\begin{aligned}
& \text { BackoffTime }= \\
& \operatorname{rand}\left[0 ; \min \left(2^{k}\left(C W_{\min }+1\right)-1, C W_{\max }\right)\right] \times \text { SlotTime, }
\end{aligned}
$$

where $k$ is the number of collisions occurred to the currently transmitted frame. The complete DCF procedure is illustrated in Fig. 1.

\section{E. Point Coordination Function}

Several QoS-aware MAC protocols utilize mechanisms similar to the Point Coordination Function (PCF) defined in IEEE 802.11. PCF provides contention-free access through a polling procedure. It was designed for infrastructure network configurations, in which one of the stations acts as a coordinator 


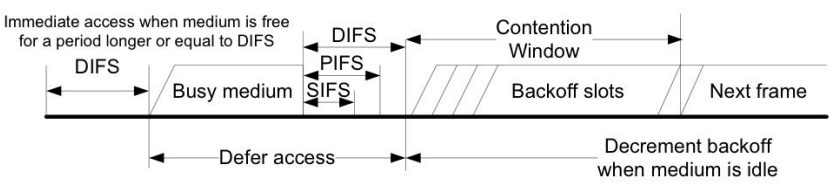

Fig. 1. DCF access method

(called the Access Point, AP) and polls other station for data. The main differences between an infrastructure network and a polling-based ad-hoc network is that in the latter any station can be selected as a network coordinator and there can be multiple such coordinators. Examples of distributed polling service-based MAC scheduling schemes can be found in [17], [18].

PCF is a centralized protocol in which stations are polled by an AP. It can operate within the same wireless network as DCF. This is achieved by alternating between the DCF and PCF access methods by using a Contention-Free Period (CFP) and a Contention Period (CP). CFP together with CP is a superframe called the Contention-Free Repetition Interval.

PCF uses a Point Coordinator (PC) operating at the AP that determines which station is assigned the right to transmit. PC selects the length of the CFP and distributes this information within Beacon management frames. This allows setting NAVs in stations within the AP range. Additionally, PCF may use the PIFS interval to give higher priority to point-coordinated traffic over DCF-controlled traffic.

Each PC maintains a polling list, that includes information about stations which support polling. In order to assign it the right to transmit, the PC selects one station from its polling list and sends a polling frame to assign the right to transmit. Each successfully transmitted frame should be acknowledged. Retransmissions of unacknowledged frames are possible during the CFP after PIFS. Because PCF is designed for single-hop networks and all transmissions are governed by the PC, the RTS/CTS exchange is not used in the CFP. The operation of PCF is illustrated in Fig. 2.

\section{Mechanisms Supported by QoS-aware MAC PROTOCOLS}

In this section we provide a novel taxonomic classification of the basic mechanisms which may be employed by MAC protocols to provide QoS in ad-hoc networks (Fig. 3). A brief explanation of the ideas behind the different mechanisms are as follows: backoff differentiation - backoff-related values are assigned according to traffic type, InterFrame Space differentiation - the waiting time required before earning the right to transmit is dependent on traffic type, jamming - pulses of energy are transmitted in order to increase the probability of successful channel access for high priority traffic, frame aggregation - data is transmitted in bursts to reduce the number of contention attempts, frame manipulation - frames with expired deadlines are dropped and/or priorities of certain frames are changed in order to expedite their transmissions, reservation predefined time periods are allocated for transmissions in order to reduce the number of collisions in the network, and alternating CP/CFP
- the channel access method alternates between contention and contention free periods.

Each mechanism included in Fig. 3 is described in more detail in the following subsections. First, a general outline of each single mechanism is provided. Additionally, in most cases, a figure illustrating the principle of operation is provided. This is followed by descriptions of protocols which utilize the given mechanism. In the first subsections, the most typical mechanisms are described: backoff/IFS differentiation, jamming, and frame aggregation/manipulation. Afterwards, two categories of periodic reservation approaches are given. Then, Alternating CP/CFP protocols, which alternate between contention and contention-free periods, are described. Finally, an overview of other unconventional approaches is provided.

\section{A. Backoff Differentiation}

Backoff differentiation is based on the idea of varying backoff-specific parameters $\left(C W_{\min }\right.$ and $\left.C W_{\max }\right)$. It is intended to give preference to high priority traffic by maximizing its wireless channel access probability (Fig. 4). The $C W_{\min }$ and $C W_{\max }$ parameters can be either fixed or variable (e.g., depending on current network saturation). In contention-based networks, backoff differentiation cannot be used to provide strict QoS.

In the literature a number of MAC protocols which employ backoff differentiation have been proposed for ad-hoc networks. However, the only standardized solution is the Enhanced Distributed Channel Access (EDCA) function (Section V-B), which is part of IEEE 802.11 and is largely based on DCF. EDCA defines four traffic priorities: Voice (Vo), Video (Vi), Best Effort (BE), and Background (BK). EDCA employs backoff procedures similar to that of DCF, but each priority has a different set of $C W_{\min }$ and $C W_{\max }$ parameters (Section V-B).

Several other QoS-aware MAC protocols use a similar or identical idea to the one used by EDCA: Modified DCF (MDCF) [19], Extended DCF with Service Differentiation [20] (supports at least two priorities), Dual-channels Request to send - RTS, Clear to send - CTS, and Ensure to send - ETS (DRCE) [21] (EDCA-like backoff is employed on a control channel), Busy Signal-based Mechanism turned On (BusySiMOn) [22]. Several other protocols modify in different ways either only the $\mathrm{CW}$ parameters or the $\mathrm{CW}$ parameters together with the DCF backoff procedure, as described below.

A priority scheme for DCF [23] introduces two nonoverlapping backoff ranges depending on the traffic priorities. For high priority traffic backoff times are chosen from the range $\left(0 ; 2^{2+i} / 2-1\right) \times$ SlotTime and for low priority traffic they are chosen from the range $\left(2^{2+i} / 2 ; 2^{2+i}-1\right) \times$ SlotTime where $i$ is the collision counter.

The Strict Priority based QoS-Aware MAC Protocol (SPQAMP) [24] also assigns non-overlapping $\mathrm{CW}$ ranges to different priorities. Additionally, if the channel is busy during the backoff timer countdown, the backoff value freezes like in DCF but only in case of real-time traffic. For best effort traffic it is reset.

In Real-time MAC (RT-MAC) [25] the CW value is proportional to the number of stations in the network. This is done 


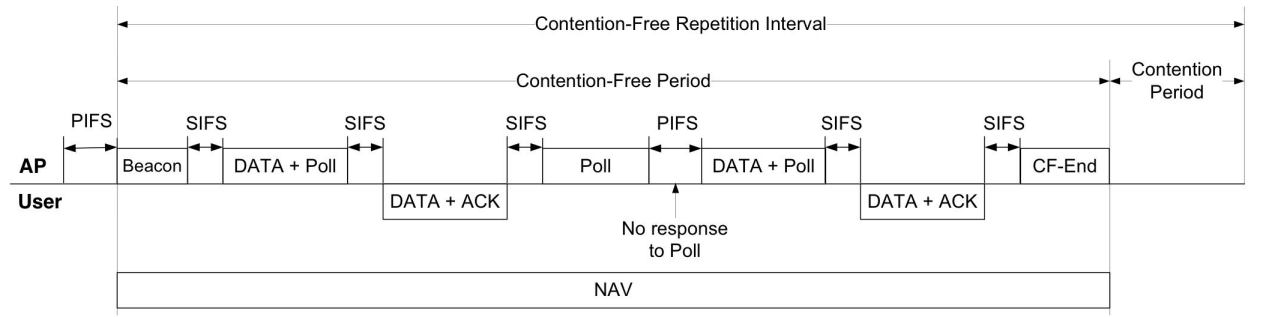

Fig. 2. PCF access method

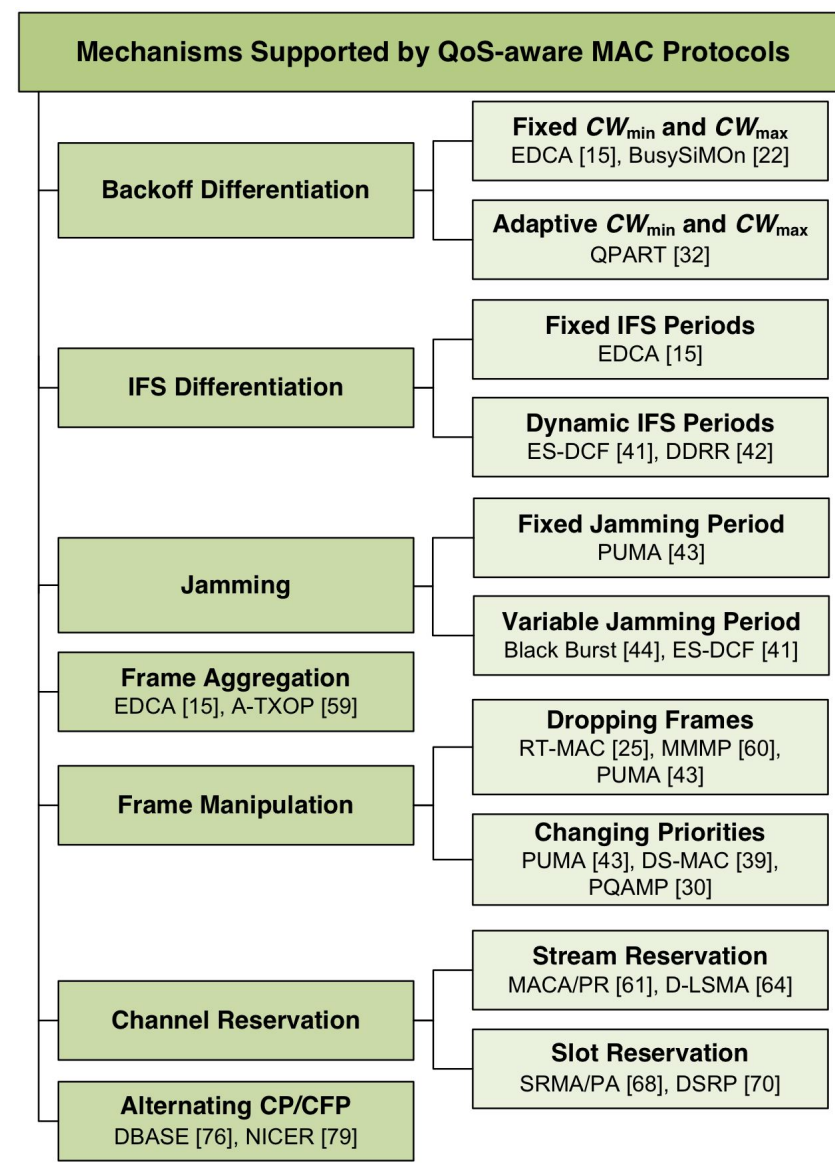

Fig. 3. Mechanisms implemented by QoS-aware MAC Protocols

in order to lower the probability of choosing the same backoff value by more than one station. To additionally eliminate the possibility of a collision, each station first selects a backoff value from the range $[0, C W-1]$, which will be used during the next transmission, and then advertises it through the header of the current frame. Stations overhearing such frames store the advertised backoff values and exclude them from their next choice of backoff.

In the Controllable Fair ID Countdown (CFIC) protocol [26] service differentiation is achieved by assigning different priority frames different backoff waiting times. In contrary to DFIC (Section IV-C) it does not cause the starvation of lower priority traffic but only delays its medium access time in comparison to high priority traffic. CFIC defines three parameters: (1) waiting unit, (2) waiting function, and (3) waiting coefficient. The waiting unit $(u)$ describes the duration of time which is the basic unit to compose the Waiting Threshold (WT) of any priority frames. The waiting function describes the relationship between $W T_{p}$ and priority level $p$ based on $u$ (i.e., it describes different waiting thresholds for different priority frames). Finally, the waiting coefficient describes the different level of differentiated service for different priority frames in the same waiting function.

The Distributed Fair Scheduling (DFS) [27] scheme combines the DCF backoff procedure with the Self-Clocked Fair Queuing (SCFQ) algorithm [28]. DFS borrows ideas from SCFQ: assigning finish and start tags to every frame arriving at each flow and transmitting a frame with the smallest finish tag. Like in SCFQ, the finish tag is inversely proportional to flow priority and directly proportional to the size of a frame. The authors propose two basic ways of calculating the backoff interval - the linear mapping scheme and the exponential mapping scheme. In the first scheme, frames with the smaller finish tags are assigned smaller backoff intervals. The backoff interval is calculated as a difference between the start and finish tags multiplied by the slot length. Additionally, to reduce the possibility of collisions backoff intervals are randomized. Finally, after a collision a new backoff value is chosen from the range $\left[1,2^{\text {CollisionCounter- } 1} \times C W\right]$, where $\mathrm{CW}$ is a constant parameter. The second scheme implements a mapping function which compresses large linear backoff intervals into a smaller exponential range. This results in reducing the time needed for the backoff countdown but may also increase the possibility of collisions. Therefore, the authors propose to use the exponential mapping scheme above a certain threshold, which is a compromise between fairness and network throughput. Additionally, the authors of DFS designed an adaptive DFS mapping scheme, which dynamically adapts DFS parameters to the changing conditions in the network. This adaptive DFS scheme is also used by the Differentiated Service-EDCA (DSEDCA) [29] protocol.

The Priority based QoS-Aware MAC Protocol (PQAMP) [30] defines four traffic priorities. For each priority, backoff intervals are chosen randomly from the range $\left[W_{i j}-\right.$ $\left.W_{j}, W_{i j}-1\right]$, where $j$ is the collision counter, $i$ is the priority, $W_{j}=2^{j} \times W_{0}, W_{i j}=(i+1) \times W_{j}$, and $W_{0}$ is the initial window size defined for DCF. This means that each traffic priority has a backoff range which does not overlap with the backoff ranges of other priorities and, additionally, the number of distinct backoff values to choose from is the same for each priority.

In the Adaptive QoS MAC Protocol (AQMP) [31] after each unsuccessful transmission the $\mathrm{CW}$ increases proportionally to a Persistence Factor (PF). CW is given by the equation: 


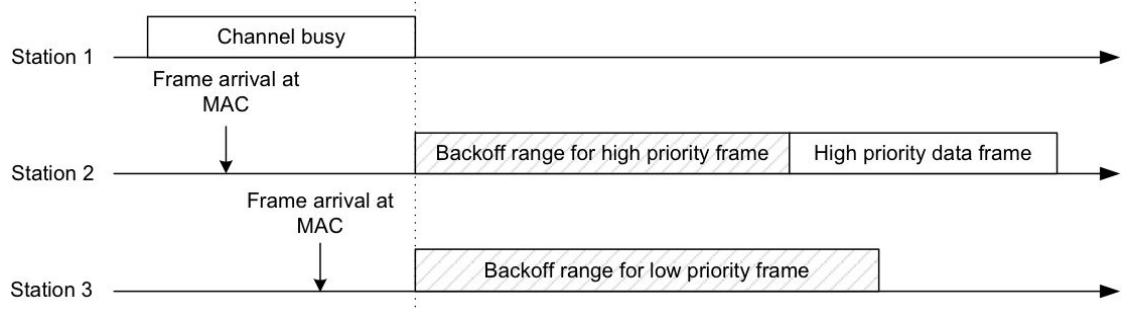

Fig. 4. Backoff differentiation

$C W_{\text {new }}=\left(C W_{\text {old }}+1\right) P F-1$, where $\mathrm{PF}$ is a function of the frame loss rate. When the current frame loss rate is lower than a previously calculated average loss rate, PF is increased for high priority traffic and decreased for low priority traffic. Otherwise, $\mathrm{PF}$ is decreased for high priority traffic and increased for low priority traffic. This assures that for large network load, high priority traffic will access the channel with a higher priority than low priority traffic. PF is within the range $\left[P F_{\min }, P F_{\max }\right]$. However, different $P F_{\min }$ and $P F_{\max }$ values are assigned to different traffic priorities.

The QoS Protocol for Ad hoc Real-time Traffic (QPART) [32] dynamically adapts $\mathrm{CW}$ sizes based on the current network congestion level and frame QoS requirements. Three types of traffic are specified: delay-sensitive, bandwidthsensitive, and best effort. A different $\mathrm{CW}$ adaptation rule is proposed for each traffic type. For delay-sensitive traffic $\mathrm{CW}$ is updated in such a way that the frame delay is kept below its per-hop delay requirement. For bandwidth-sensitive traffic $\mathrm{CW}$ is adapted by maintaining a constant queue length, which guarantees a constant value of throughput. Finally, for best effort traffic $\mathrm{CW}$ is updated in order to avoid overload situations and interfering with real-time flows.

In the Faired QoS assured MAC Protocol [33] each station along the path from the source to the destination counts the number of successful frame transmissions, separately for each flow. If two frames of the same priority but from different flows are stored in a single queue the one which belongs to the less frequently served flow can be dequeued. Such behavior is obtained by changing CWs according to a scaling factor, which is the ratio of the number of successful frame transmissions for the current flow to the number of successful frame transmissions for any better served flow of the same priority.

In [34] a modification of EDCA (Section V-B) is proposed, where the $C W_{\min }$ and $C W_{\max }$ parameters for low priority traffic are adjusted dynamically. The goal is to achieve both high network utilization and appropriate traffic differentiation. Each station measures the local network environment. If the network load is high, then the CW parameters of low priority traffic are increased to assure proper QoS parameters for high priority traffic. If the network load is low then the CW parameters of low priority traffic are decreased to achieve higher network utilization. Two data-control mechanisms are proposed for choosing the $\mathrm{CW}$ parameters: based on mapping traffic load indications to parameters or changing the parameters according to the tendency of the traffic load indications. The former provides better fairness, but obtaining the pairs of

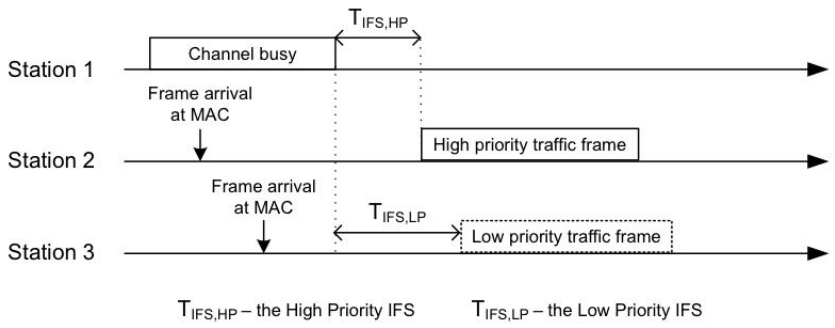

Fig. 5. Example of IFS differentiation

mapping values is difficult. The latter method is simpler and therefore better suited for practical implementation.

In [35] a linear backoff algorithm is proposed in order to decrease delay for real-time traffic. Instead of increasing CWs exponentially the authors propose changing CWs according to the equation $C W_{\text {real-time }}=\min \left(C W_{\max }, C W_{\min }(N-1)\right)$, where $\mathrm{N}$ is the number of attempts of sending that particular frame. Additionally, the authors propose reducing CWs for non-real-time traffic in stalled stations. Firstly, a special threshold value is selected which represents the maximum acceptable number of buffered data frames and a guaranteed $\mathrm{CW}$ size for the stalled stations $\left(C W_{\text {stall }}\right.$, which is smaller than $C W_{\min }$ ). If the number of buffered frames is greater than the assumed threshold, the CWs of subsequent frames are decreased to $C W_{\text {stall }}$. This helps to reduce delay of nonreal-time traffic.

\section{B. IFS Differentiation}

In CSMA/CA-based protocols, the IFS parameter designates the time required for the channel to be free before either a transmission or a backoff countdown may begin (Section III-D). MAC QoS protocols often employ diverse IFS values to provide traffic differentiation between classes (Fig. 5). Two fixed IFS times are defined: for high $\left(T_{I F S, H P}\right)$ and low $\left(T_{I F S, L P}\right)$ priority traffic. Since $T_{I F S, H P}<T_{I F S, L P}$ Station 2 is able to send its high priority frame while Station 3 has to postpone sending its low priority frame.

This method is commonly used with backoff differentiation (Section IV-A). The combination of these methods increases the probability of sending high priority before low priority frames. Therefore, relative differentiation between traffic classes is achieved. However, strict QoS guarantees on throughput and delay performance are not provided.

One of the first protocols which proposed the use of IFS differentiation in ad-hoc networks was [23]. It is a modification 
of DCF (Section III-D) which, for high priority frames, instead of DIFS utilizes PIFS (PIFS $<$ DIFS). This is possible because ad-hoc networks do not use PCF. Such an approach (i.e., choosing an IFS shorter than DIFS for high priority traffic) can ensure compatibility with legacy IEEE 802.11 stations.

Currently, the most commonplace utilization of IFS differentiation can be found in EDCA (Section V-B). In this protocol each priority has its own Arbitration Inter-Frame Space Number (AIFSN) (Section V-B). The Arbitration InterFrame Space (AIFS), which is the equivalent of DIFS, is calculated as $A I F S=S I F S+A I F S N \times T_{\text {slot }}$, where $T_{\text {slot }}$ is the length of the slot time and SIFS is the Short Inter-Frame Space (of DCF).

The authors of [36] propose a control method for selecting the AIFS parameters of EDCA. In this method only a slight difference between AIFS values is enough to ensure traffic differentiation. In [34], also based on EDCA, the AIFS parameter of low priority traffic is changed dynamically, similarly to the $\mathrm{CW}$ parameters as described in Section IV-A. Other modifications of EDCA which have been proposed in the literature (e.g., DRCE [21], [22], ETXOP [37]) adopt its IFS differentiation mechanism without changes. There also exist similar approaches tailored to specific application scenarios, e.g., for networks of Unmanned Air Vehicles (UAVs) [38] or wireless medical information systems [39].

AMP [40] employs a different AIFS differentiation, based on the frame loss rate. The idea is similar to the one used in the adaptive backoff procedure of AQMP [31]. When the frame loss rate exceeds a predefined threshold, AIFSN is decreased for high priority and increased for low priority traffic.

An alternative to combining IFS and backoff differentiation is to utilize non-fixed IFS periods and include the randomization within the IFS selection. Examples of this approach can be found in two protocols: Elimination by Sieving DCF (ESDCF) [41] and Distributed Deficit Round Robin (DDRR) [42]. In both these protocols IFS is calculated independently for each head-of-line frame based on its priority, waiting time, and a random number. This may seem like an alternative approach, however, these three conditions are a combination of multiple traffic priorities (Section V-B), frame manipulation (Section IV-D), and backoff differentiation (Section IV-A), respectively.

IFS differentiation can also be used in combination with jamming protocols (Section IV-C) [43], [44], [45], [46], or reservation-based protocols (Section IV-F) [47]. The previously described principle remains unchanged: before jamming the channel or performing a slot reservation, stations sending high priority traffic wait for the channel to be free for a shorter period than stations sending low priority traffic. In these protocols (with the exception of [44], [45]) compatibility with DCF is ensured through the use of an IFS period shorter than DIFS for high priority traffic. The opposite is true for the Distributed Priority Scheduling protocol [48], in which stations sending high priority traffic use the DIFS period while stations sending low priority traffic extend this period by a fixed amount in order to reduce contention. Finally, an interesting mathematical model to evaluate the throughput and delay performance of CSMA/CA with IFS differentiation can be found in [49].

\section{Jamming}

Jamming is based on the transmission of pulses of energy over the channel. There are a plenty of names interchangeably used in the literature to describe this operation. The most popular are: bursts, black bursts, forecast bursts, and busytones. Jamming is used to provide differentiation between traffic classes. However, different algorithms are employed to specify the length of the bursts. The most common approach is when this length is proportional to the priority (Fig. 6). After the channel has been idle for a $T_{I F S}$ period, the priority (sometimes called strict priority) is given to stations which send a jamming signal before their data transmission. The jamming and observation intervals $\left(T_{j a m}\right.$ and $\left.T_{o b s}\right)$ allow to introduce more priorities and/or to minimize the probability of collisions between stations sending frames of the same priority. Moreover, the jamming interval can be either fixed or variable (depending on the specific protocol). Jamming is usually combined with other mechanisms such as backoff or IFS differentiation to provide even better separation of traffic priorities.

The protocol which introduced the jamming technique to support real-time traffic and minimize its delay is Black Burst [44], [45]. Black Burst supports two classes of data: high priority (real-time traffic) and low priority. All high priority stations have the ability to jam the wireless channel with pulses of energy (black bursts, BBs). BBs are transmitted in the contention period: their duration is dependent on the time that the station had to wait for the channel to become idle. The more the station waited for the channel, the longer the duration of the jamming signal. Specifically, the station jams the channel using BBs for a period equal to a number of black slots. The duration of a black slot should be longer than $2 \tau$, where $\tau$ includes the maximum propagation delay, sensing delay and turnaround time of the transceiver. The number of black slots can be calculated using the formula $d / t_{\text {unit }}$, where $d$ is the time the station has been waiting for the channel access and $t_{\text {unit }}$ is a function of the observation interval, IFS time, and frame transmission time. After transmitting the jamming signal the station listens to the medium to see whether any other station is sending a longer signal. If the medium is idle, it means that the station won the contention and can proceed with data transmission. Otherwise, the station has to wait until the next contention period. The authors prove that under certain conditions each contention period guarantees a unique winner. After successful transmission of real-time frame, the station schedules the next access instant for another real-time frame. This allows for synchronization of real-time flows, and sharing the medium in a TDM-like fashion. Therefore, the Black Burst protocol can ensure bounded delay among stations sending real-time traffic.

The PUMA (Priority Unavoidable Multiple Access) protocol [43] supports strict prioritization because real-time data frames are always transmitted before non-real-time data frames. Every station that wants to start a transmission of realtime traffic sends out a jamming signal of the length of one slot if the channel has been idle for a PIFS. This signal informs stations sending non-real-time traffic, about a scheduled timebounded traffic transmission. 


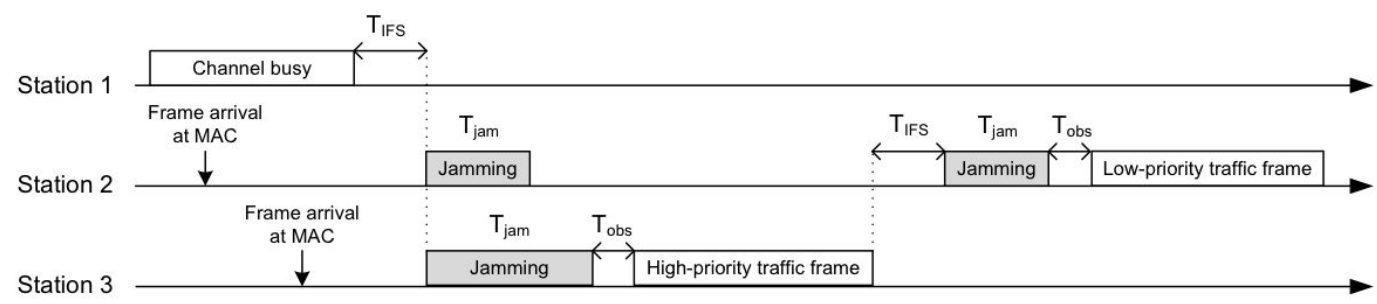

$\mathrm{T}_{\mathrm{IFS}}$ - the IFS between the next transmission

$T_{\text {obs }}$ - the observation interval

$\mathrm{T}_{\mathrm{jam}}$ - the jamming interval

Fig. 6. Example of access procedure using jamming signals

The Elimination by Sieving (ES-DCF) protocol [41] is based on DCF and incorporates some features of Black Burst [44], [45] and the Priority scheme for DCF [23]. In ES-DCF, the transmissions of BBs of the lengths equal to unique station ID numbers take place. The station that sends the longest BB wins the contention and accesses the channel at the subsequent attempt. The ES-DCF introduces the blocked-access feature, where all stations that have experienced a collision use the smallest channel-free-wait-times. This means that they can preempt all other stations during the collision resolution phase.

Deadline Bursting (DB-DCF) is the second MAC protocol proposed in [41] implementing Black Bursts. The Black Burst contention phase is, oppositely to ES-DCF, the first phase in DB-DCF. In this phase the station jams the channel with BB pulses of a length inversely proportional to the urgency of its real-time data frame. The urgency is measured in terms of the closeness to the targeted deadline. The BB lengths are integral multiples of a black slot.

In the Prioritized Binary Countdown (PBC) [50] protocol the jamming signals are transmitted as binary numbers. These numbers consist of a priority number (for QoS support) and a random number (for fairness and collision control). The frame format of the control channel is presented in Fig. 7.

In the binary countdown period the station sends a signal if the individual bit of the chosen binary number is set and remains silent if the bit is not set. The station which remains silent senses the channel. If the channel is not idle the station loses the competition. The winning station is allowed to transmit.

The priority MAC protocol to support Real-Time traffic (PMAC) [51] combines features of Black Burst [44], [45] and the randomized initialization protocol [52]. At the beginning, the BB contention is employed. Stations are divided into $m$ levels of priority. The length of a $\mathrm{BB}$ is proportional to the priority level. The station with the longest BB wins the contention. When there is more than one station of the same priority, the randomized initialization protocol is used to choose the unique winner.

The Carrier Sense Media Access with ID Countdown (CSMA/IC) [53] protocol uses two channels - control and data. CSMA/IC is used on the control channel and the data channel communication is scheduled through the control channel. In order to access the wireless channel a binary countdown mechanism in used. Each frame is assigned a binary number which is locally unique. The higher the number the higher the frame priority. Before each transmission stations send a jamming signal on the control channel, which prevents lower priority stations from transmitting data.

The Differentiated Fair ID Countdown (DFIC) mechanism [26] enhances the service differentiation of the CSMA/IC protocol by transmitting priority information at the beginning of a superframe. The DFIC mechanism supports strict prioritization. Jamming signals are used to declare frame priorities. Higher priority frames have assigned the corresponding higher digit value in the priority slots. All contending stations with lower priority frames recognize these digits and lose their rights for transmission. Therefore, only the stations with the current highest priority frames can compete for access to the channel.

The Priority-Grouped Distributed Medium Access (PGDMA) [46] employs the Black Burst mechanism before transmission of real-time traffic. The Black Burst length is proportional to a cost function that depends on the length of the scheduling phase. Therefore, frames with higher costs have longer jamming periods. The cost function can accommodate a variety of criteria such as: delay, application class, service priority, residual time-to-live constraint, etc. The authors provide several formulas for calculation of the cost function.

In the QoS-based Multiple Access (QMA) protocol [54] the channel access cycle is divided into a contention and transmission phase (Fig. 8). The contention phase is composed of $n+m$ slots which are assigned to real-time ( $n$ slots) and non-real-time traffic ( $m$ slots). Stations sending non-real-time traffic are allowed to broadcast forecast bursts (FBs) in $\mathrm{m}$ slots only when all $\mathrm{n}$ slots are idle. This assures priority of real-time traffic over non-real-time traffic. A station chooses a random number $b$ using a truncated geometric distribution. If a station senses the first $b$ slots idle, it immediately starts transmission of $k \times$ FB slots. Otherwise, it freezes its backoff. The $k$ parameter depends on the frame lifetime. The frame with the earliest deadline has the largest $k$ value. After transmission of FBs the station senses the medium. If the channel is busy, it means that there must be at least one contending station with a higher priority frame, and the station with a lower priority frame loses the competition. Otherwise, if the channel is idle, the station can start its transmission. Summarizing, the station which sends the largest number of FBs wins the overall 


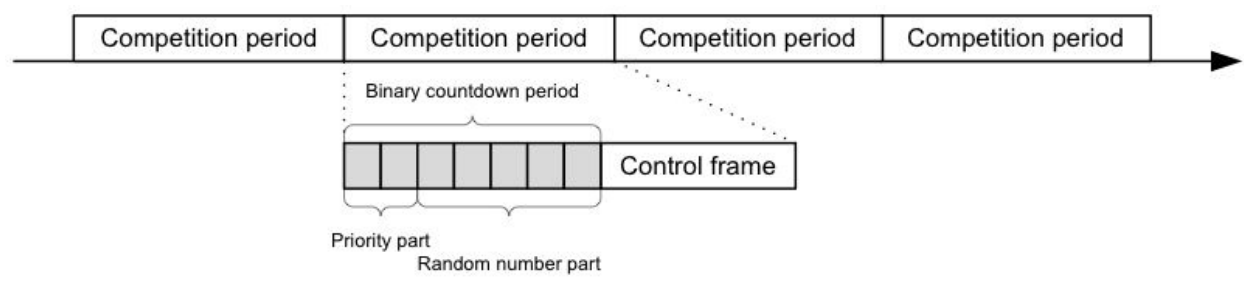

Fig. 7. The frame format for the control channel of PBC

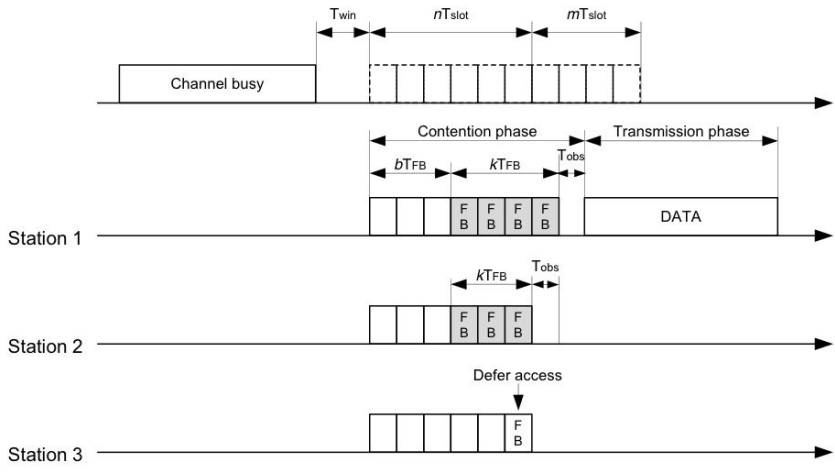

Fig. 8. Access procedure in QMA

contention. The QMA protocol guarantees that only stations that start transmission of FBs in the same slot can successfully survive the contention phase.

Wang at. all proposed a new busy-tone-based MAC protocol that supports voice and non-QoS data traffic in non-fully connected ad-hoc networks [55]. The proposed MAC protocol similarly to DBTMA [56] utilizes two narrow-band busy tone channels and one information channel. The station starts to send a busy tone in the transmitter busy tone channel, where the duration of the busy tone equals its backoff timer. Next, the station senses this channel again, and if the channel is idle, the station can start its transmission. The voice frames are sent immediately after the station finishes its busy tone transmission. It is also assumed that RTS is always transmitted before sending an asynchronous data frame. The busy tone transmitted by the receiver in the receiver busy tone channel, serves as the ACK and CTS frames.

BusySiMOn [22] combines the idea of jamming with the advantages of EDCA traffic differentiation. It employs two types of busy tone signals. The first type is used by a sender station to preliminary reserve the wireless channel. The second type is used by its neighboring stations to confirm the reservation as well as to inform possible hidden stations about the planned transmission. Both busy tone signals are very short in comparison to legacy control frames (RTS and CTS), which increases the probability of successful channel reservation and assures better QoS support.

\section{Frame Aggregation}

The frame aggregation mechanism (also referred to: packettrain, frame bursting, frame grouping, contention-free burst, transmission opportunity) allows a single station to send a series of uninterrupted frames. This reduces the contention overhead. Frame aggregation is perceived as a technique that increases the overall network throughput but it should be used very carefully in order to maintain fair use of the medium by multiple stations. It is usually used to support voice or video transmissions where a large number of short frames are generated by the source. This allows for transmission of more real-time frames per time interval at the cost of wait time for users sending non-real-time traffic. Frame aggregation is especially useful when there are only a few stations contending for access to the channel (e.g., point-to-point links). The typical access procedure using the frame-bursting mechanism is presented in Fig. 9.

The station that wins the contention (has the smallest backoff value in TB) starts transmission of frames from its queue without relinquishing control of the transmission medium. To increase the protocol efficiency, all continuously transmitted frames are separated with a smallest defined time interval $\left(T_{\text {short }}\right)$. The frame aggregation protocols usually define a limit, on the time duration or number of frames that can be transmitted in such a manner. The frame acknowledgement policy can be either No-ACK (no expected acknowledgement to reduce the protocol overhead), Block-ACK (one ACK frame for the whole burst) or separate ACK for each frame in the burst.

The original idea of frame aggregation in wireless networks was proposed in the FAMA protocol [57], however the first QoS-aware protocol that uses this technique is PUMA [43]. This mechanism is used in PUMA only for the transmission of real-time traffic. PUMS uses No-ACK policy.

IEEE 802.11 proposed a solution for EDCA called TXOP (Transmission Opportunity), in which stations are allowed to transmit continuously several frames [15]. The standard defines TXOP as a time interval when a station has the right to initiate transmission of frame(s) in sequence onto the wireless medium. This prevents stations transmitting frames with low rate from gaining access to the channel for an excessive amount of time (as it was in DCF). The TXOP is characterized by a starting time and a maximum duration. When the station obtains a TXOP, it can transfer at least the first frame waiting in its transmission queue. Each access category has a maximum channel occupancy time, called $T X O P_{\text {Limit }}$ which is a configurable parameter. This parameter also depends on the physical layer type as defined in [15]. A $T X O P_{\text {Limit }}$ equal to 0 implies that only one frame can be transmitted after the station obtains access to the channel. When the $T X O P_{\text {Limit }}$ is greater than zero, the station is allowed to transmit as long as the total channel occupancy time is less or equal than the $T X O P_{\text {Limit }}$. Additionally, EDCA employs the Block-ACK policy. The same frame aggregation mechanism is used in derivatives of EDCA, such as EDCA/RR [58]. 


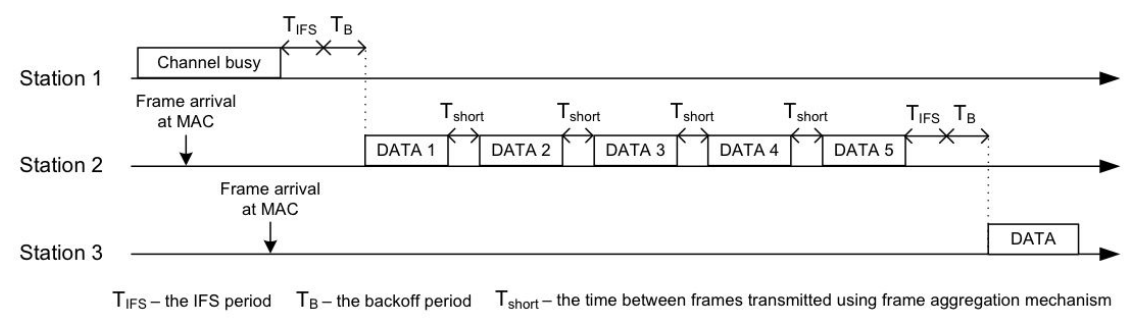

Fig. 9. Example of a frame aggregation mechanism (with No-ACK)

The Adaptive-TXOP (A-TXOP) [59] protocol decreases the transmission delay by reducing the number of channel contentions required to transmit large video frames (e.g., I frames in MPEG streams). The authors propose to modify the TXOP interval dynamically based on the frames in the queue, so that fragments of the same frame can be sent in the same TXOP interval. In contrast to video traffic, a single voice flow usually has low bandwidth requirements and cannot fully utilize a single TXOP burst. Moreover, in a multihop environment an intermediate station will have to forward frames of several flows and so will have frames to several stations in its queue. Therefore, the newly proposed TXOPsharing mechanism allows reducing the delay of voice traffic by using the TXOP to transmit to multiple stations, in order to fully utilize the TXOP interval. Therefore, it reduces the number of channel contentions and thereby reduces the delay of voice traffic.

The Enhanced Transmission Opportunity (ETXOP) protocol [37] is a protocol based on EDCA which employs dynamical assignment of $T X O P_{\text {Limit }}$ values for high priority traffic. These TXOP Limit values are calculated according to its priority (intra-QoS differentiation) and flow data rate (inter-QoS differentiation). Low priority traffic has static $T X O P_{\text {Limit }}$ values.

\section{E. Frame Manipulation}

Several protocols implement frame manipulation techniques in order to maximize network throughput and provide better QoS. They either employ dropping frames with expired deadlines or changing frame priorities to increase their chances of successful delivery. The description of both techniques is given next.

1) Dropping Frames: Frame dropping can be used to discard frames which have expired. This requires the addition of deadlines to delay-sensitive frames. The expiration check can occur either: (i) during the preparation for transmission, after backoff timer expiry, and after each unsuccessful transmission (RT-MAC [25]), (ii) when the isochronous frame is removed from the MAC queue, after counting the backoff timer, and after receiving the CTS frame (PUMA [43]), (iii) after frames arrive at the MAC layer and before they are sent from the MAC layer to the PHY layer (RACSS [38]), or (iv) at the start of each transmission attempt and just before the transmission of an RTS frame (ES-DCF and DB-DCF [41]).

An alternative frame dropping mechanism for multimedia traffic is defined by MMMP [60]. It is based on the observation that video traffic (e.g., MPEG-4) is structured in specific groups. When the first frame in a group (i.e., I frame) is lost, subsequent frames (i.e., $\mathrm{P}$ frames) are useless. Therefore, a whole group of frames is discarded if the whole I frame or only its part was lost. Additionally, expired frames are dropped.

2) Changing Priorities: The goal of changing priorities is to increase the probability of successful delivery of particular frames and decrease their delay. In the literature several different approaches are proposed to meet this goal.

In DPS [48] the priority of a frame changes dynamically when it traverses the path towards the destination. Every time when a frame suffers from an excessive upstream queuing delay, its priority is increased by the downstream stations. This allows the frame to reach the destination station before its deadline expires.

PUMA [43] uses a special timer that determines the nonreal-time frame timeout. If a non-real-time frame reaches its life-time it is transmitted as a real-time frame. This allows proper scaling and contention of both types of traffic.

In [59] the authors noticed that frames which have to traverse a large number of hops rarely meet their deadlines. Therefore, they propose to give higher priority to such frames but without starving other frames. To meet this goal, stations store in their queues two non-standard parameters: frame deadline and the remaining number of hops to the destination. Additionally, dedicated ReAllocative Priority (ReAP) schedulers decide on the order of frame transmissions in each of the queues. Similarly, PQAMP [30] gives higher priority to relayed frames than to locally generated ones. This allows to minimize delays on multi-hop paths.

\section{F. Channel Reservation}

There are two categories of methods for performing periodic reservations: stream and slot reservation. Both require some form of reservation tables, however, the former is designed strictly for real-time traffic while the latter can be applied to any traffic profile.

1) Stream Reservation: To reduce the number of contention attempts for sensitive real-time data a number of stream reservation protocols based on CSMA/CA have been proposed. Their principle of operation is illustrated in Fig. 10. For non-real-time data the channel access is contention-based. For real-time data, however, there exist designated reserved periods for every real-time stream (or call) generated by a station. To prevent multiple stations from transmitting during a reserved period, each station maintains a continuously updated reservation table (RT) with the scheduled transmissions. Realtime frames are not retransmitted. The stream reservation 


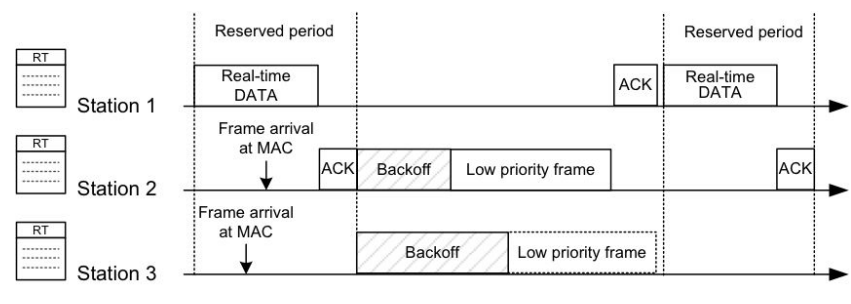

RT - Reservation Table

Fig. 10. Example of a stream reservation protocol

approach is best suited for real-time streams but not for other types of high priority traffic, which may not be periodic and may not tolerate packet loss.

MACA/PR [61] (based on DCF) is a prominent example of a stream reservation protocol. In this protocol it is assumed that real-time frames arrive at constant time intervals. This assumption allows for the reservation periods to be evenly spaced for each stream. The initial reservation of the transmission period is performed (through an RTS/CTS exchange) when the first real-time frame is sent. This reservation is done for every link on the multi-hop path between the source and the destination. ACK frames contain scheduling information in their headers and thus renew the reservation. The reservation is released when the reserved period has been determined idle for a given time. The RTs are periodically shared between stations to prevent conflicts. Standard DCF is used for nonreal-time frames. There is a limit on the maximum number of reservations to prevent non-real-time frames from being denied channel access.

Several direct modifications of MACA/PR have been proposed in the literature. They extend it by providing the following features: separate transmit/receive reservation tables [60], [62], resource reservation based on stream bandwidth requirements [60], bandwidth reallocation [60], and power control [63].

D-LSMA [64] extends the stream reservation concept by introducing five categories of time periods in the reservation table: free (open for reservation), reserved (neither transmission nor reception is allowed), transmit OK (only transmission is allowed), receive OK (only reception is allowed), and semireserved (the RTS/CTS exchange has not been completed). This detailed scheduling approach solves the exposed station problem and allows for higher network utilization. Additionally, the reservation can be performed for all data types (not only real-time streams).

The EDCA with Resource Reservation (EDCA/RR) protocol [58] allows to reserve TXOPs for high priority traffic streams. EDCA/RR introduces the traffic specification (TSPEC) element which contains information about traffic characteristics and QoS expectations. In order to determine whether the new stream can be admitted, the scheduled service interval (SI) and service start time (SST) parameters are calculated. As shown in Fig. 11, SI is the interval between TXOPs. Frame aggregation is used within a TXOP (Section IV-D).

An add traffic stream (ADDTS) frame contains a TSPEC element (which includes SI and SST) and is broadcasted to schedule the new traffic stream. All stations store information

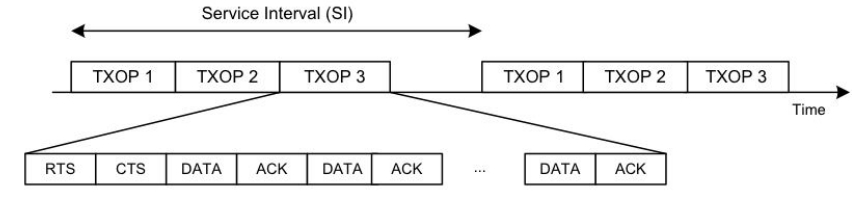

Fig. 11. Sequence of frames in EDCA/RR

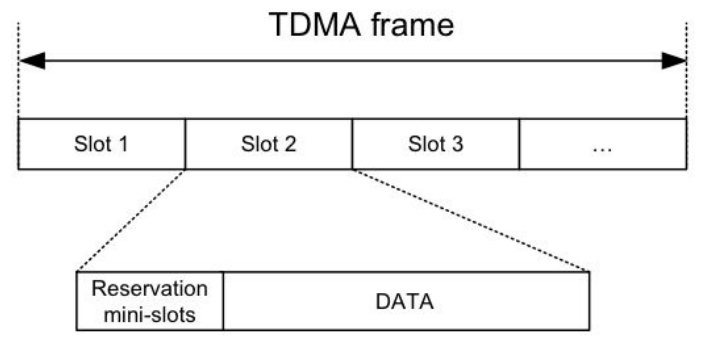

Fig. 12. Example of a slot reservation protocol

of the sender's SST and SI, and schedule the new traffic stream exactly as the sender. This ensures collision-free access to the medium to streams with reserved TXOPs. The station is allowed to transmit frames from other traffic categories by contending for access to the medium, however, the station must finish its transmission before a next reserved TXOP starts. When a transmission failure occurs during a TXOP, the station tries to retransmit the failed frame after SIFS if there is enough time left in the TXOP to finish the transmission.

Furthermore, several other protocols employ a stream reservation mechanism: MAC-FM introduces the possibility to transmit stream frames in non-reservation periods to avoid scheduling collisions [65], C-MAC decreases overhead by having the destination send the CTS after the preliminary reservation [35], the New MAC Protocol with Pseudo-TDMA Behavior enables stream reservation by utilizing EDCA-based backoff and IFS differentiation as well as admission control [66], and the IEEE 802.11 MAC protocol for Multi-hop Reservation extends RTS frames with additional information (e.g., the destination IP address) and forwards them to perform a multi-hop reservation to the destination [67].

2) Slot Reservation: Slot reservation protocols apply TDMA, instead of CSMA/CA, to access the channel (Fig. 12). However, some CSMA/CA mechanisms, such as RTS/CTS are also present here. Time is divided into periodic TDMA frames, each containing a fixed number of equal size slots. At the beginning of each slot stations attempt to perform a reservation. Slot reservations continue in each TDMA frame until the end of transmission. Additionally, slot reservation protocols require time synchronization, e.g., by a special synchronization field at the beginning of each slot or by a global clock (such as from GPS). This synchronous approach usually has higher complexity but can achieve better utilization and give fixed delay which is important for real-time traffic. Difficulties for ad-hoc networks include frequent topology changes which can disturb the meticulously set up slot reservations.

The Soft Reservation Multiple Access with Priority Assignment (SRMA/PA) protocol [68] is a good example of 
a slot reservation protocol and one of the first such protocols proposed for ad-hoc networks. Two traffic classes are distinguished: real-time and best effort. Each frame, based on its class, is given an initial priority level. This level directly determines the frame's probability of reserving a slot. An RTS/CTS-like handshake at the beginning of every idle slot is used for this reservation. Additionally, before this handshake, there is a designated mini-slot which allows a realtime frame to take away the reservation from a best effort frame. Contention within a priority level is resolved using a backoff procedure. However, the backoff occurs on whole slots (Fig. 12) and not on short periods as in DCF.

In D-PRMA [69] slots can be either used for sending DATA frames (as presented in Fig. 12) or for reservation. Such reservation slots consist of multiple mini-slots in which the scheduling of future frames is determined. An RTS/CTSlike exchange is used to determine which station wins the contention. Two rules are used to prioritize traffic: (1) in the first mini-slot high priority traffic can always contend while low priority can contend only with a certain probability and (2) high priority traffic can reserve the same slot in subsequent TDMA frames while low priority traffic always has to contend for the slot. The winner of the first minislot may use the remaining mini-slots for transmission of its data. A similar approach is used in DSRP [70]. Again, the probability of sending an RTS for high priority traffic is higher than for sending lower priority traffic. However, only high priority traffic can compete in the first mini-slot. Additionally, DSRP employs a frame priority changing mechanism based on time limits (Section IV-E2). QoS-Croma [71] also uses an RTS/CTS-like exchange in the reservation mini-slots (Fig. 12). The difference is that RTS is sent only during initial reservation. In subsequent TDMA frames, the transmission is destination-initiated through CTS. This polling approach allows the receiver to communicate with multiple senders during a given slot in consecutive TDMA frames. Furthermore, an Earliest Deadline First (EDF) [72] scheduling algorithm is applied by the receiver to determine which stations to poll so that QoS guarantees are met. These QoS parameters (i.e., requested and available bandwidth) are exchanged in RTS/CTS frames.

The Dynamic TDMA Slot Reservation (DTSR) protocol [73] is designed for ad-hoc networks which utilize cognitive radio technology. The slot reservation approach is applied to reserve both the slot and the channel. Reservation and control information is sent during a designated slot at the beginning of the TDMA frame while the remainder of the TDMA frame consists of DATA/ACK exchange slots. Interestingly, the TDMA frame length can be changed dynamically according to the number of stations and their bandwidth requirements.

Similarly to DTSR, Throughput-aimed MAC (T-MAC) [74] is also a $\mathrm{QoS}$ protocol utilizing cognitive radio and is based on a TDMA approach. In T-MAC the cyclic TDMA frame consists of reservation slots, information slots (for DATA) and acknowledgement slots (for ACK). The reservation is performed using a three-way handshake in the reservation slots. Each reservation slot has several corresponding information slots and a single acknowledgement slot. This approach protects the ACK frames. Additionally, the RTs include transmission

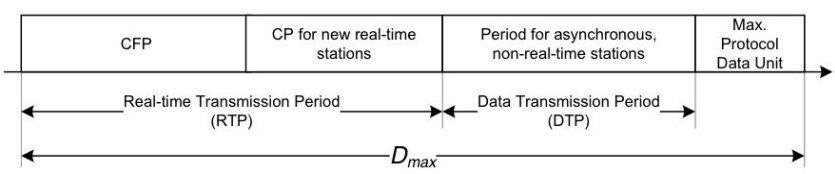

Fig. 13. Superframe structure of the DBASE protocol

power information to facilitate concurrent transmissions.

In the Seedex protocol [75] the problem of slot assignment is solved through reservation tables and a pseudo-random number generator. In each slot a station may either listen (state $L$ ) or possibility transmit (state $P T$ ). This is determined by a pseudo-random number generator. Therefore, stations need only exchange the generator seeds between them. A transmission occurs with probability $p$ when the sender is in $P T$ and destination is in $L$. ACK frames are used to confirm correct reception of DATA frames. Finally, to provide traffic differentiation, $p$ can be adjusted for high and low priority traffic.

\section{G. Alternating $C P / C F P$}

Alternating $\mathrm{CP} / \mathrm{CFP}$ protocols incorporate two different access methods in a single MAC protocol: a contention-free and a contention-based method. The former usually supports transmission of real-time traffic while the latter supports best-effort traffic (this also includes the transmission of control frames used for reserving the contention-free period). Contentionfree access can be realized in a number of ways, however three methods are especially important: TDMA, polling and token-based. Contention-based access can also use a number of QoS mechanisms such as: jamming and backoff or IFS differentiation. The operation of alternating CP/CFP protocols is similar to the concept of PCF (Section III-E), as showed in Fig. 2.

The key idea of Distributed Bandwidth Allocation/Sharing/Extension (DBASE) protocol [76] is that each station maintains a Reservation Table, which stores information about reservations for real-time traffic transmissions. As illustrated in Fig. 13, in DBASE time is divided into the Real-time Transmission Period (RTP) and the Data Transmission Period (DTP).

The repetition period $D_{\max }$, also known as the superframe, cannot be larger than the maximum tolerable delay for realtime traffic. RTP consists of CFP and contention period for new real-time traffic. The latter is a period in which stations reserve the channel. The first active real-time station in the adhoc is a coordinator. It periodically broadcasts a reservation frame RTS at the beginning of each CFP. This frame contains information about all reservations stored in the RT. The reservation procedure is based on the RTS/CTS exchange. Subsequent frames belonging to the real-time session are sent in CFP. DTP is intended for non real-time traffic and the access procedure in DTP is based on DCF. The maximum Protocol Data Unit (PDU) period following DTP is added to allows the completion of any remaining transmissions, which started very late in the DTP period.

In Mobile Point Coordinator for MAC (MPC-MAC) [77] the infrastructure is created dynamically. Any station can be 
selected to be a Mobile Point Coordinator (MPC), which plays the role of an access point in the ad-hoc environment. In contrast to a typical infrastructure-based network there can be multiple MPCs, within each others' communication range. These MPCs poll other stations during the CFP. The process of MPC selection is based on the periodical transmission and reception of "hello" messages by all stations. If the received signal is stronger than the MPC candidate threshold parameter, then the station is within the destination station's MPC range and this station can be put on its list of the possible MPC candidates. After collecting information about neighboring stations for a predefined time interval, the station selects an MPC from its list of possible candidates. The station can also select itself as an MPC candidate. To solve possible conflicts between multiple MPCs, a Slot Defer Number (SDN) mechanism is proposed. This mechanism extends the PIFS period to several time slots, as in the IEEE 802.11 DCF, to avoid collision among the MPCs and stations. There can also be several PCF initiators co-existing together in the MPC, which need to share a superframe time period. Therefore, there may be several CFP and CP periods in a superframe, fragmented and interleaved together.

Frame switching based on PCF protocol presented in [78] is based on a hierarchical approach consisting of two sub layers. The lower layer provides a fundamental access method based on DCF to support non-real-time data traffic and enables time sensitive traffic to reserve bandwidth using a two-way handshake mechanism. The upper layer uses a combined $(\mathrm{DCF}+\mathrm{PCF})$ mode to support real-time periodic traffic. Each station acts as a coordinator. This means that each station is responsible for maintaining its own polling-list to regulate and schedule the transmissions. Additionally, the protocol assumes two types of channels: common (in which all stations can use to disseminate and acquire neighbor- and routing-related information) and unique (where each station has a unique and orthogonal code used to receive frames from others). The receiver-based spread-spectrum technique is used to accommodate simultaneous transmission of several data frames using multiple parallel channels (created with different spreading codes). If a real-time traffic needs to be transmitted in the network with hidden stations, through the intermediate station, the source station has to send an "Association Request" frame using the contention period. Intermediate station has to answer with an "Association Response" frame to the source station also during the contention period. Moreover, intermediate station has to create a polling-list and include source station in it. Such a station is called virtual PC (VPC), and at the start of the contention free period, station intermediate has to begin polling of source station. Intermediate station should repeat the whole procedure and send the "Association Request" frame to a destination station. A new IFS called Reservation IFS (RIFS) was defined (PIFS $<$ RIFS $<$ DIFS) for real-time traffic. It is used before the "Association Request" frame transmission, while DIFS is used to obtain access for non-real-time traffic. Admission control module located at every station analyzes the available resources before sending the "Association Response" frame. The maximum number of real-time traffic that can be supported in CFP is limited to provide proper QoS guarantees.

The Neighborhood Information with Class Estimation and
invited-Reservation (NICER) protocol [79] employs an invited reservation procedure and a distributed frame synchronization mechanism. Similarly to other alternating CP/CFP protocols, NICER uses the concept of superframe. The role of the coordinator is held by all receivers of real-time traffic. To assure proper reservations for real-time traffic, NICER consists of a few procedures. The observation procedure allows stations to record information about all types of transmitted traffic (also from other stations). Two separate tables are defined for every station to $\log$ the reservation information of real-time traffic and properties of non-real-time traffic flows. The contention procedure allows for transmission of non-real-time traffic and making reservations for real-time traffic transmissions in the CFP. A linear backoff algorithm is used before the transmission of the first frame of real-time traffic (only for the reservation purposes) in the $\mathrm{CP}$. In the invited reservation procedure all receivers of real-time traffic should send in the CFP a real-time clear-to-send (rt-CTS) control frame, to invite the source stations to send real-time data frames. This procedure allows to avoid collisions of real-time traffic and also resolves the hidden station problem.

R-CSMA/CA [47] introduces a Reservation Coordination Function (RCF) which supports real-time periodic traffic through slot reservations prior to the actual data transmission. This protocol also employs the superframe concept, however instead of PCF, the RCF is proposed on top of DCF to support real-time periodic traffic. R-CSMA/CA assumes that there is no central coordinator and every station should participate in reservation procedures. A distributed algorithm based on the three-way handshaking is utilized to support slot reservations. Therefore, the $\mathrm{CP}$ is used to send both non-realtime traffic and to make reservations for TDMA slots. An RCF-IFS (RIFS) interval (DIFS $<$ RIFS $<$ SIFS), is used in the $\mathrm{RCF}$ function to make a slot reservation for real-time traffic. Apart from RTS, CTS and ACK control frames, the protocol adds three new frames: RFS (Request for Slot Reservation), RAC (Reservation Acknowledgement) and RAN (Reservation Announcement). The purpose of the RFS frame is to inform about available slots from the perspective of the source station and request for a reservation. If there is an available slot in the destination station's table, the destination station responds with an RAC frame and confirms the reservation. Then, the source station uses a RAN frame to announce the slot reservation to the neighboring stations.

MAC-FM(E) [65] enhances the MAC-FM protocol [65] for a large number of stations sending real-time traffic. With the growth of the number of such stations, the delay in MAC-FM increases. To solve this problem MAC-FM(E) introduces a periodic superframe. Furthermore, CFP is divided into Reserve and Data Transfer period. All control frames are transmitted in the Reserve period to reserve and release time slots in the Data Transfer period. The Data Transfer period is used to transfer data frames. The length of the Reserve period and the position in the superframe is constant unlike the length of Data Transfer period, which depends on the throughput of the real-time service. Additionally, there is no single coordinator and every station should maintain an RT. This table can also be used to specify the beginning of each $\mathrm{CP}$. 


\section{H. Other Mechanisms}

This section describes protocols which employ non-typical approaches and cannot be easily assigned to any of the abovementioned categories.

The Distributed Differentiated Scheduling (DDS) mechanism [80] detaches control frames from DATA frames by using maximum reservation lag times, which are dependent on frame priorities. The maximum reservation lag time value is included in the RTS and CTS frames. High priority frames are assigned maximum reservation lag times which allow avoiding competition with low priority frames at the receiver. This mechanism is also employed in Multiple Access with Lag Time (MALT) [81] and Advance Access (AA) [82] protocols.

The Clustering-based Multichannel MAC protocol [83] groups stations into clusters based on their mobility. A contention-free TDMA-based MAC protocol is used within a cluster to provide deterministic delay. A contention-based protocol is used for communication between clusters. Additionally, multiple channels are used with high priority traffic having a separate channel.

DS-MAC [39] introduces a preemptive scheduling algorithm which allows a higher priority frame to interrupt an ongoing transmission of a lower priority frame if both frames are transmitted by the same station. After a successful transmission of all higher priority frames the interrupted frames are resumed.

The Hybrid Token-CDMA MAC protocol (HT-CDMA) [84] is based on a token-passing protocol and CDMA. The token-passing approach ensures contention-free access to the medium, while CDMA supports multiple simultaneous transmissions. In order to provide QoS guarantees, a modified leaky-bucket algorithm is implemented: each station in HTCDMA is assigned a specific traffic class. The number of frames that can be transmitted is dependent on the traffic class. In HT-CDMA, a single token is created for the network and $M$ codes are generated and distributed by means of the token. The token is passed in a predefined order among the stations (i.e., each station maintains a table of its neighbors and knows where to pass the token). Stations are equipped with two transceivers - one for data transmission and one for passing the token. One of the most important fields of the token is the Number Of Codes (NOC) parameter. The NOC value specifies how many codes are used within the network. When a particular code is released, NOC is decremented by 1 . Otherwise, if the station captures the token and starts a transmission, NOC is incremented by 1 . The token is forwarded to the successor before the station starts its data transmission, which reduces the token rotation time and improves utilization of bandwidth. HT-CDMA introduces a unique feature - Packet Collision Avoidance (PCA) algorithm. According to PCA, the Channel List field in the token is checked before transmission to verify whether the destination is able to receive data.

The Multi-Channel Token Ring Protocol (MCTRP) [85] for inter-vehicle communications implements adaptive ring coordination and channel scheduling. It assumes that all vehicles are equipped with two radios and that system is synchronized. Vehicles are automatically organized into multiple virtual rings, each using a different channel, depending on their velocity and road traffic conditions. Communication is possible thanks to inter- and intra-ring message exchange. The inter-ring data communication is realized using CSMA/CA and the intra-ring data communication is realized using tokenbased data exchange. Finally, MCTRP employs additional three protocols for: ring coordination, emergency message exchange between rings, and intra-ring data exchange.

The QoS Cooperative Stream Controlled Medium Access (QCSCMA) [86] mechanism employs the Multiple Input Multiple Output (MIMO) technique to simultaneously transmit several data streams on a single link. Each station maintains a table with QoS parameters of stations within a two-hop range. These QoS parameters are piggybacked in modified RTS and CTS frames. On the basis of this information, a sender station decides on a particular link on which its DATA frames will be transmitted. It also decides on the number of streams which will be used for transmission. The authors of QCSCMA protocol consider only long-term QoS requirements of individual flows.

The Wireless Real-Time Ring (WRT-Ring) [87] uses CDMA mechanism to allow multiple transmissions without causing collisions. Therefore, each station in the network is assigned a unique code. Additionally, in order to communicate with all other stations, each station uses a common code (broadcast channel representation). WRT-Ring requires stations to form a virtual ring. After ring initialization, a TDMA mechanism is used, which divides the communication channel into slots. Each slot is divided into a header and a data part. The former indicates the status of the current slot (busy or empty), the latter may contain data. Additionally, a control signal (SAT) circulates in the ring. It gives a predefined number (equal to $l$ for real-time traffic and $k$ for best effort traffic) of transmission opportunities to each station. After SAT leaves a station, the station can transmit up to $l$ realtime frames and $k$ best effort frames.

The Wireless Token Ring Protocol (WTRP) [88] is a contention-free protocol that guarantees bounded delay and fair share of bandwidth. The protocol assumes coexistence of multiple rings and channels in the network. Stations choose which channels to join using information included in the token. In each ring there is a limited number of stations. If there are multiple nearby token rings, a channel allocator is used to allocate channels to achieve spatial reuse, which maximizes the network capacity. Additionally, each ring has a unique ring owner that allows the stations to distinguish between messages coming from different rings. WTRP employs also several management functions. It utilizes a mobility manager that decides when a station should join or leave the ring and an admission control manager that limits the number of stations that have permission to transmit on the wireless channel in order to assure them bounded delay and reserved bandwidth. The time sequence diagram of the WTRP is shown in Fig. 14. After each data frame transmission, stations send tokens to their successors. Furthermore, WTRP specifies the maximum latency that a member station of a ring can tolerate. In case when the token is lost (e.g., when the station discovers that its successor is unreachable) a ring recovery mechanism is invoked.

In [89] a multi-channel protocol was proposed. It operates in 


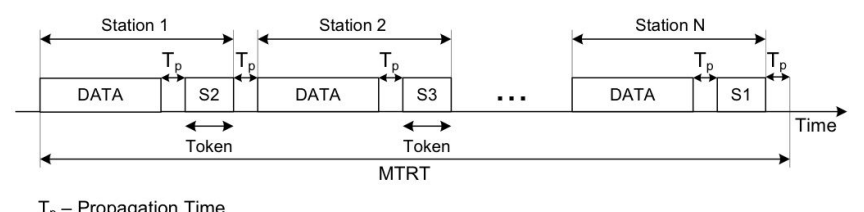

Fig. 14. Time diagram of WTRP [88]

two phases: control and data transmission. The control phase is divided into two parts: backoff-based contention of real-time traffic users and backoff-based contention of data traffic users. The contention of real-time traffic users is realized before the contention of data traffic users. Additionally, the protocol employs three control frames: RTS, CTS, and Reservation (RES). Control frames are used to negotiate the channel number for data transmission. In the data transmission phase each station transmits on the chosen channel. The proposed protocol is a work in progress and, therefore, many important details about its operation are still missing.

QoS-aware Slotted-Aloha based UWB MAC with Cooperative Retransmissions (SAUCeR) [90] utilizes slotted-aloha to reduce collisions and, therefore, it requires time synchronization of stations. Two ultimate goals of SAUCeR are improvement of the overall network throughput and reduction of end-to-end delay. It is a distributed MAC protocol in which the frame structure presented in Fig. 15 is used.

The DATA part of the time frame is divided into $\mathrm{m}$ slots. Differentiated QoS is provided by allocating separate time slots to different traffic classes. Therefore, high and low priority traffic is transmitted in randomly selected slots within the High and Low Priority CW, respectively. The CW values are dynamically changed, depending on the amount of traffic belonging to each of the traffic classes, in order to prevent starvation of either traffic class. Additionally, SAUCeR implements a QoS-aware cooperative retransmission technique. Each station maintains two buffers: data (with locally generated DATA frames) and overhearing (with successfully overheard DATA frames). DATA frames stored in either buffer can be transmitted during the DATA part of a time frame. After a positive acknowledgement they are removed from their buffers. Finally, two distributed relay selection protocols are introduced (based on highest signal strength and random selection), which help to select an appropriate relay after a DATA frame was overheard by more than a single station.

\section{Standardized Protocols}

This section describes three standardized MAC protocols which provide QoS in ad-hoc networks: the legacy EY-NPMA for HIPERLAN networks, the IEEE 802.11 EDCA, and the MCCA from the upcoming IEEE 802.11s standard for mesh networks. ${ }^{2}$ All these protocols adopt mechanisms previously described in Section IV. This section concludes with an account of ongoing standardization work in the field of QoS MAC protocols for ad-hoc networks.

\footnotetext{
${ }^{2}$ Mesh networks differ from ad-hoc networks in that they have designated immobile nodes (mesh routers) which form the backbone infrastructure. They retain the multi-hop nature of ad-hoc networks and therefore the QoS challenges are similar.
}

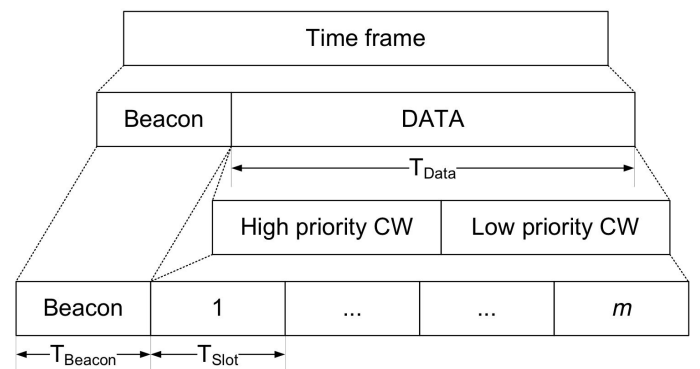

Fig. 15. SAUCeR frame structure

\section{A. EY-NPMA}

Elimination-Yield Non-Pre-emptive Priority Multiple Access (EY-NPMA) is the MAC protocol of the ETSI HIPERLAN standard [91]. It supports traffic differentiation via hierarchically independent priorities. EY-NPMA employs CSMA technique (referred to the standard as the synchronized channel condition). It uses several access cycles to obtain a very low collision rate by reducing the number of active stations in each cycle. EY-NPMA utilizes timestamp-based frame dropping (Section IV-E1) and dynamic assignment of access priorities based on the remaining lifetime of the frames. Depending on its residual lifetime, the frame is assigned one of the five priorities, with 0 being the highest priority. There are four channel access phases: the prioritization, elimination, yield and data transmission phase (Fig. 16).

In the prioritization phase a station, with a channel access priority $n$, should listen for $n$ prioritization slot intervals. If the channel is sensed idle in the $n$-th prioritization slot interval, the station transmits a channel access burst of the duration of a priority assertion interval. Only stations which have the highest channel access priority move to the next phase. Therefore, in the next, elimination phase, only stations with the same, highest priority contend for channel access. Each station selects a random number of slots, which follows a truncated geometric distribution. Next, the station transmits these slots as a channel access burst. As soon as a station finishes its transmission, it checks the medium. If the medium is busy it defers. If it is idle, the station can proceed to the yield phase. There is the possibility that two or more stations having the same priority frame will choose the same length of elimination pulse, and be unable to detect one another even at the end of the second phase. In this case the final contention is done in the yield phase. The yield phase is similar to the backoff procedure used by IEEE 802.11 DCF. EY-NPMA assures strict QoS guarantees and time bounded delivery of frames. It is also the basis of the Siren MAC architecture [92].

\section{B. $E D C A$}

The IEEE 802.11 EDCA protocol defines several extensions to the DCF access method. It introduces four access categories (ACs): Vo, Vi, BE, and BK (Fig. 17). Each AC has its own set of channel access parameters which are used to provide traffic differentiation and described below. Note that EDCA is the only QoS protocol which has been implemented in commercial IEEE 802.11 cards by numerous vendors. 


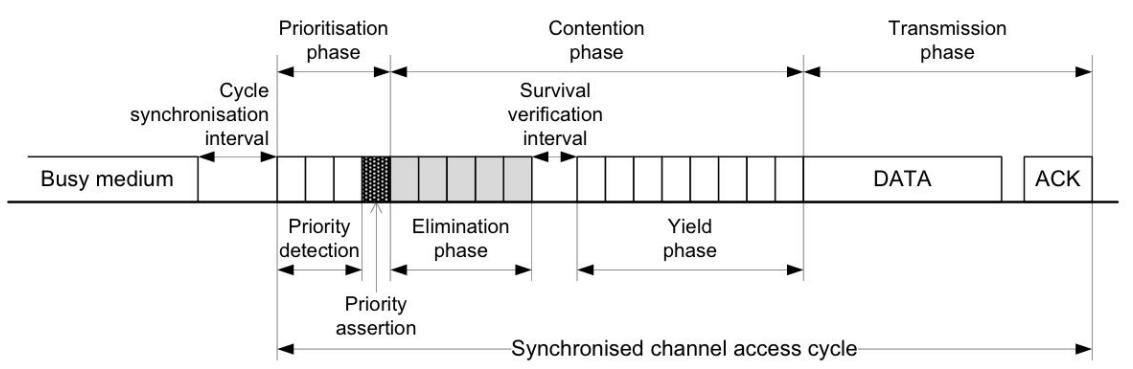

Fig. 16. EY-NPMA channel access cycles

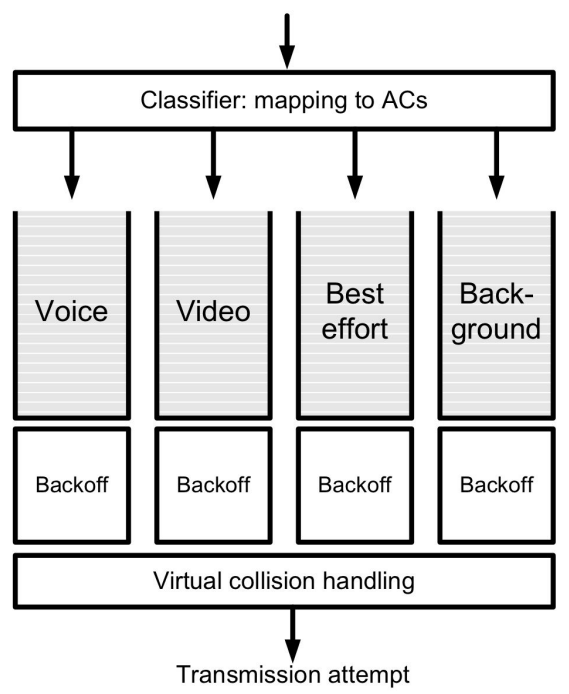

Fig. 17. EDCA backoff procedure

The process of channel access prioritization is depicted in Fig. 18 and can be briefly described as follows. Frames are buffered into one of four hardware transmission queues. After that, the probability of being granted the channel access is dependent on four access parameters: Arbitrary Interfame Space Number (AIFSN[AC] Section IV-B), Contention Window minimum and maximum values $\left(C W_{\min }[A C]\right.$ and $C W_{\max }[A C]$ Section IV-A), and an optional Transmission Opportunity

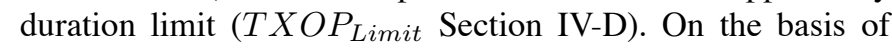
access parameters the values of the backoff interval and AIFS are computed for each of the ACs. The backoff value is set to a random natural number from the interval $[0, C W]$, where $\mathrm{CW}$ initially equals $C W_{\min }[A C]-1$ and is increased up to $C W_{\max }[A C]$ whenever the $\mathrm{AC}$ involves in a collision. Once the $C W_{\max }[A C]$ is reached, retries will continue until a maximum number of retries allowed is reached. AIFS[AC] is given by equation: $A I F S[A C]=S I F S+A I F S N[A C]$ SlotTime.

A QoS station is assigned the right to transmit every time when the medium was sensed idle for the whole AIFS[AC] interval and when the backoff time for a particular $\mathrm{AC}$ has elapsed. As a consequence, the smaller the AIFSN[AC] and the current $\mathrm{CW}$ size, the higher the probability of being granted earlier access to the wireless medium.

A virtual collision occurs when more than one $\mathrm{AC}$ within a station is granted the right to transmit at the same time. In that case, the frame from the highest priority queue involved in the collision is chosen and transmitted, while the lower priority frames undergo the backoff procedure. All the mechanisms

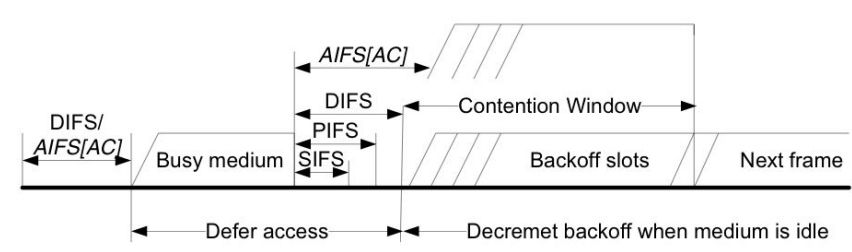

Fig. 18. Channel access prioritization

utilized by EDCA ensure traffic differentiation but cannot provide strict QoS guarantees.

\section{C. $M C C A$}

In the recently published (September 2011) IEEE 802.11s standard [93] medium access for wireless mesh networks is governed by the Mesh Coordination Function (MCF), which is a hybrid approach consisting of a contention-based period (governed by EDCA, Section V-B) and a contention-free period (governed by Mesh Coordinated Channel Access, MCCA) [94]. MCCA is a distributed slot reservation protocol (Section IV-F2) designed for communication within the multi-hop backbone mesh network. The time frame (Fig. 12) is divided into fixed slots. Mesh stations can reserve as much consecutive slots for transmission (MCCA opportunities, MCCAOPs) as required to send a DATA frame. The reservation is set up (and tore down) using control messages and its duration is based on traffic characteristics. To decrease the number of collisions the MCCAOPs are advertised in beacons within a two-hop range. The station with the MCCAOP uses EDCA (e.g., with zero backoff) for transmission. Therefore, it can still collide with non-MCCA stations. However, MCCA should prove to be a valid solution for multi-hop transmissions in static mesh networks.

\section{Ongoing Standardization Work}

The IEEE 802.11 standard is constantly being extended. Currently there are two standardization groups which are active in areas directly related to QoS: 802.11aa (Medium Access Control Enhancements for Robust Audio Video Streaming) [95] and 802.11ae (Prioritization of Management Frames) [96]. The former amendment specifies the following enhancements to the MAC layer: (i) smooth degradation of audio video streams in case of insufficient channel capacity, (ii) more robust performance of overlapping BSS environments, (iii) intraaccess category prioritization of streams by modifying EDCA timing/parameters, (iv) improved reliability and jitter characteristics for multicast and broadcast audio video streams, 
TABLE I

COMPARISON OF MAC QOS MECHANISMS

\begin{tabular}{|c|c|c|c|c|}
\hline Mechanism & QoS Approach & Advantages & Disadvantages & Coexistence with Legacy 802.11 \\
\hline Backoff differentiation & Prioritized & $\begin{array}{l}\text { Easy to implement, common approach, stan- } \\
\text { dardized [15], provides contention resolu- } \\
\text { tion }\end{array}$ & $\begin{array}{l}\text { Less strict prioritization than with } \\
\text { IFS differentiation }\end{array}$ & Yes \\
\hline IFS differentiation & Prioritized & $\begin{array}{l}\text { Easy to implement, common approach, stan- } \\
\text { dardized [15], supports strict prioritization }\end{array}$ & Prone to high overhead & Yes \\
\hline Jamming & Prioritized & $\begin{array}{l}\text { Easy to implement, bursts are easy to detect, } \\
\text { supports strict prioritization }\end{array}$ & $\begin{array}{l}\text { High energy consumption, prone to } \\
\text { high overhead }\end{array}$ & Yes \\
\hline Frame aggregation & Prioritized & $\begin{array}{l}\text { Reduces contention overhead and long term } \\
\text { number of contention attempts }\end{array}$ & $\begin{array}{l}\text { Appropriate only for certain traffic } \\
\text { types (short frames), introduces ag- } \\
\text { gregation delay }\end{array}$ & Yes \\
\hline Dropping frames & Prioritized & Reduces congestion and delay & Applicable to certain traffic types & $\mathrm{N} / \mathrm{A}$ \\
\hline Changing priorities & Prioritized & $\begin{array}{l}\text { Increases probability of successful frame } \\
\text { delivery, decreases delay }\end{array}$ & Applicable to certain traffic types & N/A \\
\hline Stream reservation & Parameterized & $\begin{array}{l}\text { Bounded throughput and delay, reduces } \\
\text { number of contention attempts }\end{array}$ & $\begin{array}{l}\text { Large signaling overhead } \\
\text { (exchange of reservation tables), } \\
\text { applicable to certain traffic types }\end{array}$ & No \\
\hline Slot reservation & Parameterized & $\begin{array}{l}\text { Bounded throughput and delay, high chan- } \\
\text { nel utilization }\end{array}$ & $\begin{array}{l}\text { Very complex (reservation tables } \\
\text { and synchronization), not suitable } \\
\text { for high mobility scenarios }\end{array}$ & No \\
\hline Alternating $\mathrm{CP} / \mathrm{CFP}$ & Both & $\begin{array}{l}\text { Allows separation of periodic and bursty } \\
\text { traffic }\end{array}$ & $\begin{array}{l}\text { Very complex, coordination re- } \\
\text { quired for CFP }\end{array}$ & No ${ }^{3}$ \\
\hline
\end{tabular}

and (v) interworking with 802.1Qat, 802.1Qav, and 802.1AS standards. The $802.11 \mathrm{ae}$ amendment defines mechanisms for prioritizing IEEE 802.11 management frames in a way which does not negatively impact application performance. Even though the size of signaling frames is usually small they can still impede the performance of applications if they are sent with high priority and the management traffic is frequent. To summarize, these activities of the IEEE 802.11 standardization groups prove that QoS in wireless networks remains an area of future work.

\section{COMPARISONS}

In this section, we first compare all the QoS mechanisms described in the survey and then proceed to examine the protocols which implement these mechanisms. Table I summarizes the advantages and disadvantages of the described QoS mechanisms. Additionally, the mechanisms are grouped according to the two QoS approaches: prioritized and parameterized (defined in Section III-A). The last column in this table shows whether stations employing a given mechanism can communicate with legacy IEEE 802.11 stations (i.e., using DCF). This is an indication of how difficult it would be to implement the mechanism using commercially available equipment. Regarding the QoS issues listed in Section III-C, it can be noticed that all the surveyed mechanisms provide support for multiple traffic types and deal with the lack of centralized coordination in accessing a shared channel. Therefore, it can be concluded that addressing these two particular issues is a very general definition of a QoS mechanism for ad-hoc networks. Additionally, it can be determined (based on Table I) that mechanisms which provide support for application requirements necessitate complex signaling and synchronization.

To provide a basis for comparing the QoS-aware MAC protocols described in this survey, they have been summarized in Table II and Table III. The former presents a description of each protocol while the latter contains information about the supported QoS mechanisms. The first columns in Table II provide basic information about each protocol: name, ref- erence, and year of publication. Protocol characteristics are also described in the table: strict priority support (SPS) - the ability to strictly guarantee channel access for high priority frames over low priority frames, traffic classes (TCs) - the total number of traffic classes supported, the extensibility of the number of traffic classes (ETC) - the possibility to add additional traffic classes, and the supported RTS/CTS reservation $(\mathrm{R} / \mathrm{C})$. The subsequent three columns ( $\mathrm{SO}-$ the size of the signaling overhead, QoS RT - QoS reservation time, OCRT - overall channel reservation time) provide an assessment of the protocol performance. This assessment is based either in comparison to EDCA or, if such a comparison was impossible, on our best technical judgment. The table entries should serve only as an approximation and guideline for readers. Furthermore, also note that the values in these columns do not necessarily validate the performance (throughput, delay, jitter) of the protocol but rather its behavior. The final column in this table provides additional comments about the protocol, e.g., support for additional features not mentioned in the other columns.

\section{Analysis of the ScIENTIFIC PRoduction on QoS MECHANISMS FOR AD-HOC NETWORKS}

The detailed comparison of the QoS mechanisms presented in Tables II and III allows us to draw several interesting conclusions. In Fig. 19 we present the number of protocols as a function of the number of supported QoS mechanisms. It can be concluded that a typical QoS-aware protocol for ad-hoc networks uses only one QoS mechanism. The more QoS mechanism supported, the higher the protocol complexity, and the lower the number of available protocols. Only five protocols presented in this survey simultaneously use four different QoS mechanisms, and none of them uses five or more such mechanisms.

\footnotetext{
${ }^{3}$ In most cases methods which alternate $\mathrm{CP} / \mathrm{CFP}$ periods are incompatible with legacy IEEE 802.11 devices. However, if a protocol implementing this method behaves similarly to PCF (Section III-E) then such coexistence can be achieved.
} 
TABLE II: Comparison of QoS-aware MAC protocols

(SPS - strict priority support, TCs - number of supported traffic classes, R/C - RTS/CTS Reservation, ETC - extensibility of the number of traffic classes, SO - size of the signaling overhead, QoS RT - QoS reservation time, OCRT - overall channel reservation time)

\begin{tabular}{|c|c|c|c|c|c|c|c|c|c|c|}
\hline No. & Protocol & Year & SPS & TCs & ETC & $\mathbf{R} / \mathbf{C}$ & SO & QoS RT & OCRT & Comments \\
\hline 1 & Black Burst [44] [45] & 1996 & Yes & 2 & No & No & Large & Slow & Slow & $\begin{array}{l}\text { Signaling overhead depends on the } \\
\text { number of stations sending real- } \\
\text { time traffic }\end{array}$ \\
\hline 2 & MACA/PR [61] & 1997 & Yes & 2 & No & Yes & Medium & Fast & Fast & $\begin{array}{l}\text { First stream reservation proto- } \\
\text { col, limited number of real-time } \\
\text { streams can be admitted, also de- } \\
\text { fines a QoS routing protocol }\end{array}$ \\
\hline 3 & EY-NPMA [91] & 1998 & Yes & 5 & Yes & No & Large & Medium & Slow & $\begin{array}{l}\text { Solves the hidden station problem, } \\
\text { assures very low collision rate }\end{array}$ \\
\hline 4 & $\begin{array}{l}\text { Priority scheme for DCF } \\
{[23]}\end{array}$ & 1999 & No & 4 & Yes & No & Medium & Medium & Medium & $\begin{array}{l}\text { First protocol with backoff and IFS } \\
\text { differentiation }\end{array}$ \\
\hline 5 & RT-MAC [25] & 1999 & No & 2 & No & No & Medium & Medium & Medium & $\begin{array}{l}\text { QoS is not provided per frame pri- } \\
\text { ority but rather per station }\end{array}$ \\
\hline 6 & M-DCF [19] & 2000 & No & 2 & Yes & No & Medium & Medium & Medium & $\begin{array}{l}\text { Data terminals utilize the silence } \\
\text { period of voice communication }\end{array}$ \\
\hline 7 & SRMA/PA [68] & 2000 & Yes & 2 & No & Yes & Medium & Slow & Slow & First slot reservation protocol \\
\hline 8 & $\begin{array}{l}\text { Extended DCF with Ser- } \\
\text { vice Differentiation [20] }\end{array}$ & 2001 & No & 2 & Yes & Yes & Medium & Medium & Medium & $\begin{array}{l}\text { Includes a passive monitoring } \\
\text { scheme is also proposed }\end{array}$ \\
\hline 9 & Seedex [75] & 2001 & No & $>1$ & $\overline{Y e s}$ & $\overline{\text { Yes }}$ & Medium & Fast & Fast & $\begin{array}{l}\text { Seeds of pseudo-random number } \\
\text { generator are exchanged }\end{array}$ \\
\hline 10 & DBASE [76] & 2001 & Yes & 2 & No & $\overline{\text { Yes }}$ & Medium & Medium & Slow & $\begin{array}{l}\text { Real-time traffic with CBR and } \\
\text { VBR characteristics is supported, } \\
\text { every station must overhear reser- } \\
\text { vation frames }\end{array}$ \\
\hline 11 & PUMA [43] & 2002 & Yes & 2 & No & Yes & Medium & Slow & Medium & $\begin{array}{l}\text { Always uses RTS/CTS, solves the } \\
\text { hidden station problem, includes } \\
\text { traffic scaling, frame aggregation, } \\
\text { and the DIDD backoff [97] mech- } \\
\text { anisms }\end{array}$ \\
\hline 12 & ES-DCF [41] & 2002 & Yes & 2 & No & No & Large & Slow & Slow & $\begin{array}{l}\text { Signaling overhead depends on the } \\
\text { number of stations sending real- } \\
\text { time traffic, solves the hidden sta- } \\
\text { tion problem }\end{array}$ \\
\hline 13 & DB-DCF [41] & 2002 & Yes & 2 & No & No & Large & Slow & Slow & $\begin{array}{l}\text { Signaling overhead depends on the } \\
\text { number of stations sending real- } \\
\text { time traffic, solves the hidden sta- } \\
\text { tion problem }\end{array}$ \\
\hline 14 & DDRR [42] & 2002 & No & $>1$ & Yes & No & Medium & Medium & Medium & $\begin{array}{l}\text { Randomness in IFS instead of } \\
\text { backoff }\end{array}$ \\
\hline 15 & DPS [48] & 2002 & No & $>1$ & Yes & Yes & Large & Medium & Slow & $\begin{array}{l}\text { Forwarded frames priority is based } \\
\text { on delay }\end{array}$ \\
\hline 16 & D-PRMA [69] & 2002 & No & 2 & No & Yes & Medium & Slow & Slow & $\begin{array}{l}\text { First mini-slot winner may use re- } \\
\text { maining mini-slots for data trans- } \\
\text { mission }\end{array}$ \\
\hline 17 & PBC [50] & 2003 & Yes & $>1$ & Yes & Yes & Large & Medium & Slow & $\begin{array}{l}\text { Requires a control channel, solves } \\
\text { the hidden station problem, de- } \\
\text { signed for multi-hop ad-hoc net- } \\
\text { works, requires dual channel }\end{array}$ \\
\hline 18 & AA [82] & 2003 & No & 4 & Yes & Yes & Medium & Slow & Slow & $\begin{array}{l}\text { Solves the hidden and exposed sta- } \\
\text { tion problems, requires dual chan- } \\
\text { nel, introduces separation between } \\
\text { RTS/CTS and DATA transmissions }\end{array}$ \\
\hline 19 & MALT [81] & 2003 & No & 4 & Yes & Yes & Medium & Slow & Slow & $\begin{array}{l}\text { Power-aware, requires dual chan- } \\
\text { nel, introduces separation between } \\
\text { RTS/CTS and DATA transmissions }\end{array}$ \\
\hline 20 & DDS [80] & 2003 & No & $>1$ & Yes & Yes & Medium & Slow & Slow & $\begin{array}{l}\text { Separation of RTS/CTS and DATA } \\
\text { transmissions }\end{array}$ \\
\hline 21 & WRT-Ring [87] & 2003 & No & 2 & Yes & No & Small & Fast & Fast & Utilizes CDMA \\
\hline 22 & CSMA/IC [53] & 2003 & No & $>1$ & Yes & No & Medium & Slow & Slow & $\begin{array}{l}\text { Requires synchronization and a } \\
\text { dual channel }\end{array}$ \\
\hline 23 & $\begin{array}{l}\text { IFS Based Service Differ- } \\
\text { entiation [36] }\end{array}$ & 2003 & No & 4 & Yes & Yes & Medium & Fast & Fast & $\begin{array}{l}\text { Relies on controlling the length of } \\
\text { the IFS interval }\end{array}$ \\
\hline 24 & MMACA/PR [62] & 2003 & Yes & 2 & & $\overline{\text { Yes }}$ & Large & Fast & Medium & $\begin{array}{l}\text { Separate transmit/receive reserva- } \\
\text { tion tables }\end{array}$ \\
\hline 25 & NICER [79] & 2003 & $\overline{\text { Yes }}$ & 2 & No & No & Small & Fast & Medium & Solves the hidden station problem \\
\hline 26 & P-MAC [51] & 2004 & Yes & $>1$ & $\overline{\text { Yes }}$ & No & Large & Medium & Slow & $\begin{array}{l}\text { Adds multiple priorities to the } \\
\text { BlackBurst protocol, requires col- } \\
\text { lision detection }\end{array}$ \\
\hline 27 & RACSS [38] & 2004 & No & $>1$ & Yes & Yes & Small & Medium & Fast & $\begin{array}{l}\text { Support for receiver-initiated trans- } \\
\text { missions }\end{array}$ \\
\hline
\end{tabular}




\begin{tabular}{|c|c|c|c|c|c|c|c|c|c|c|}
\hline 28 & R-CSMA/CA [47] & 2004 & Yes & 2 & No & Yes & Large & Slow & Slow & $\begin{array}{l}\text { Reservations performed in the con- } \\
\text { tention period }\end{array}$ \\
\hline 29 & $\begin{array}{l}\text { Local data-control } \\
\text { schemes [34] }\end{array}$ & 2004 & No & 4 & Yes & No & Medium & Medium & Medium & $\begin{array}{l}\text { Backoff differentiation based on } \\
\text { traffic load indications }\end{array}$ \\
\hline 30 & D-LSMA [64] & 2004 & Yes & 2 & No & Yes & Large & Medium & Medium & Very detailed reservation tables \\
\hline 31 & QPART [32] & 2004 & No & $>1$ & Yes & No & Medium & Medium & Medium & $\begin{array}{l}\text { Adaptive } \mathrm{CW} \text { based on passive } \\
\text { congestion monitoring }\end{array}$ \\
\hline 32 & WTRP [88] & 2004 & No & 1 & No & Yes & Small & Fast & Fast & $\begin{array}{l}\text { Guarantees bounded latency and } \\
\text { reserved bandwidth }\end{array}$ \\
\hline 33 & MPC-MAC [77] & 2004 & Yes & 2 & No & Yes & Medium & Medium & Slow & $\begin{array}{l}\text { Several CP/CFP periods can exist } \\
\text { within a superframe, uses a polling } \\
\text { list }\end{array}$ \\
\hline 34 & $\begin{array}{l}\text { Frame switching based on } \\
\text { PCF [78] }\end{array}$ & 2004 & Yes & 2 & No & Yes & Medium & Medium & Slow & $\begin{array}{l}\text { Uses receiver-based spread- } \\
\text { spectrum technology, admission } \\
\text { control and polling list, adapts } \\
\text { PCF to ad-hoc networks }\end{array}$ \\
\hline 35 & DFIC [26] & 2004 & Yes & $>1$ & Yes & No & Medium & Medium & Slow & $\begin{array}{l}\text { High priority traffic can completely } \\
\text { starve low priority traffic }\end{array}$ \\
\hline 36 & CFIC [26] & 2004 & No & $>1$ & Yes & No & Medium & Slow & Slow & Prone to misconfiguration \\
\hline 37 & EDCA [15] & 2005 & No & 4 & Yes & Yes & Medium & Medium & Medium & Standardized QoS MAC protocol \\
\hline 38 & DRCE [21] & 2005 & No & 4 & Yes & Yes & Large & Medium & Medium & $\begin{array}{l}\text { Employs power control, requires } \\
\text { dual channel }\end{array}$ \\
\hline 39 & DFS [27] & 2005 & No & $>1$ & Yes & No & Medium & Medium & Medium & $\begin{array}{l}\text { QoS is provided per flow, frames } \\
\text { with the smallest finish tags are } \\
\text { transmitted first }\end{array}$ \\
\hline 40 & ETXOP [37] & 2005 & No & 4 & Yes & No & Medium & Medium & Medium & $\begin{array}{l}\text { Supports inter- and intra-QoS dif- } \\
\text { ferentiation, includes admission } \\
\text { control }\end{array}$ \\
\hline 41 & PGDMA [46] & 2006 & Yes & $>1$ & Yes & Yes & Large & Slow & Slow & $\begin{array}{l}\text { Transmissions of prioritized frames } \\
\text { in a TDMA fashion, solves the } \\
\text { hidden station problem }\end{array}$ \\
\hline 42 & MAC-FM [65] & 2006 & Yes & 2 & No & Yes & Large & Medium & Medium & $\begin{array}{l}\text { Allows real-time frames in con- } \\
\text { tention periods }\end{array}$ \\
\hline 43 & $\begin{array}{l}\text { New MAC Protocol with } \\
\text { Pseudo-TDMA Behavior } \\
\text { [66] }\end{array}$ & 2006 & No & 2 & No & Yes & Large & Medium & Medium & Includes admission control \\
\hline 44 & EDCA/RR [58] & 2006 & Yes & 4 & Yes & Yes & Medium & Medium & Slow & $\begin{array}{l}\text { Combines EDCA } \\
\text { reservation }\end{array}$ \\
\hline 45 & MAC-FM(E) [65] & 2006 & Yes & 2 & No & Yes & Large & Medium & Slow & $\begin{array}{l}\text { CP is used for real-time traffic and } \\
\text { CFP is used for best effort traffic }\end{array}$ \\
\hline 46 & QMA [54] & 2007 & Yes & 2 & No & No & Large & Slow & Slow & $\begin{array}{l}\text { Intensive real-time traffic can com- } \\
\text { pletely starve best effort traffic }\end{array}$ \\
\hline 47 & MMMP [60] & 2007 & Yes & 2 & No & Yes & Large & Medium & Medium & $\begin{array}{l}\text { Bandwidth reservation based on } \\
\text { stream requirements, possible } \\
\text { bandwidth reallocation }\end{array}$ \\
\hline 48 & ReAllocative Priority [59] & 2007 & No & 4 & Yes & Yes & Medium & Medium & Medium & $\begin{array}{l}\text { Non-standard schedulers, adaptive } \\
\text { TXOP }\end{array}$ \\
\hline 49 & MPPC [63] & 2007 & Yes & 2 & No & Yes & Large & Medium & Medium & $\begin{array}{l}\text { Separate transmit/receive reserva- } \\
\text { tion tables, includes power control }\end{array}$ \\
\hline 50 & C-MAC [35] & 2007 & Yes & 2 & Yes & Yes & Large & Medium & Medium & Utilizes cognitive radio \\
\hline 51 & DS-EDCA [29] & 2007 & Yes & 4 & Yes & Yes & Medium & Large & Large & $\begin{array}{l}\text { Provides strict priority and } \\
\text { weighted fair service among } \mathrm{ACs}\end{array}$ \\
\hline 52 & DSRP [70] & 2007 & Yes & 2 & No & Yes & Medium & Slow & Slow & $\begin{array}{l}\text { Only high priority traffic can com- } \\
\text { pete in the first mini-slot }\end{array}$ \\
\hline 53 & QoS-Croma [71] & 2007 & No & $>1$ & Yes & Yes & Medium & Medium & Medium & Receiver-initiated \\
\hline 54 & $\begin{array}{l}\text { Adaptive-TXOP } \\
\text { TXOP) [59] }\end{array}$ & 2007 & No & 4 & Yes & Yes & Medium & Medium & Medium & $\begin{array}{l}\text { Improves the Frame Delivery Ratio } \\
\text { of multimedia traffic }\end{array}$ \\
\hline 55 & $\begin{array}{l}\text { Clustering-based } \\
\text { Multichannel } \\
{[83]}\end{array}$ & 2007 & Yes & 2 & No & Yes & Medium & Medium & Slow & $\begin{array}{l}\text { Supports seven separate channels, } \\
\text { requires two transceivers, uses } \\
\text { CDMA codes }\end{array}$ \\
\hline 56 & DS-MAC [39] & 2008 & Yes & 3 & Yes & Yes & Medium & Medium & Medium & $\begin{array}{l}\text { Designed for medical information } \\
\text { systems, low priority transmissions } \\
\text { can be interrupted by high priority } \\
\text { transmissions }\end{array}$ \\
\hline 57 & PQAMP [30] & 2008 & No & 4 & Yes & Yes & Medium & Medium & Medium & $\begin{array}{l}\text { Each traffic priority has a backoff } \\
\text { range which does not overlap with } \\
\text { the backoff ranges of other priori- } \\
\text { ties }\end{array}$ \\
\hline 58 & MCTRP [85] & 2008 & Yes & 2 & No & No & Medium & Medium & Medium & $\begin{array}{l}\text { Alternates between token (low pri- } \\
\text { ority) and CSMA/CA periods (high } \\
\text { priority) }\end{array}$ \\
\hline 59 & MCCA [93] & 2008 & No & 4 & Yes & Yes & Large & Slow & Slow & $\begin{array}{l}\text { Standardized MAC protocol for } \\
\text { mesh networks }\end{array}$ \\
\hline 60 & HT-CDMA [84] & 2008 & No & $>1$ & Yes & No & Medium & Fast & Medium & $\begin{array}{llll}\text { Uses } & \text { CDMA, } & \text { requires } & \text { two } \\
\text { transceivers }\end{array}$ \\
\hline
\end{tabular}




\begin{tabular}{|c|c|c|c|c|c|c|c|c|c|c|}
\hline 61 & $\begin{array}{l}\text { Busy-tone-based MAC } \\
{[55]}\end{array}$ & 2008 & Yes & 2 & No & Yes & Large & Medium & Slow & $\begin{array}{l}\text { Uses two busy-tone channels with } \\
\text { different carrier sensing ranges and } \\
\text { one information channel, solves the } \\
\text { hidden and exposed station prob- } \\
\text { lems }\end{array}$ \\
\hline 62 & SPQAMP [30] & 2008 & Yes & 2 & Yes & Yes & Medium & Medium & Medium & $\begin{array}{l}\text { A strict non-overlapping range of } \\
\text { contention windows are assigned to } \\
\text { real-time and best-effort traffic }\end{array}$ \\
\hline 63 & AQMP [31] & 2009 & No & $>1$ & Yes & No & Medium & Medium & Medium & $\begin{array}{l}\text { Stations withhold from transmis- } \\
\text { sion if queue loss is high, solves } \\
\text { the hidden station problem }\end{array}$ \\
\hline 64 & BusySiMOn [22] & 2010 & No & 4 & Yes & Yes & Medium & Fast & Medium & $\begin{array}{l}\text { Includes TXOP, solves the hidden } \\
\text { station problem }\end{array}$ \\
\hline 65 & QCSCMA [86] & 2010 & No & $>1$ & Yes & Yes & Medium & Medium & Medium & Employs MIMO \\
\hline 66 & AMP [40] & 2010 & No & $>1$ & Yes & No & Medium & Medium & Medium & Similar to AQMP \\
\hline 67 & $\begin{array}{l}\text { DTSR [73] } \\
\end{array}$ & 2010 & No & 1 & No & Yes & Large & Medium & Large & $\begin{array}{l}\text { Supports dynamic frame length, } \\
\text { utilizes cognitive radio }\end{array}$ \\
\hline 68 & T-MAC [74] & 2010 & No & 1 & No & Yes & Large & Medium & Large & Utilizes cognitive radio \\
\hline 69 & $\begin{array}{l}\text { Multi-channel MAC with } \\
\text { service differentiation [89] }\end{array}$ & 2011 & Yes & 2 & Yes & Yes & Medium & Medium & Medium & $\begin{array}{l}\text { Channel reservation phase com- } \\
\text { posed of two parts: contention of } \\
\text { real-time users and contention of } \\
\text { data traffic users involves multiple } \\
\text { channels }\end{array}$ \\
\hline 70 & FQSA-MAC [33] & 2011 & No & 3 & Yes & No & Medium & Medium & Medium & $\begin{array}{l}\text { The number of successful frame } \\
\text { transmissions is calculated on the } \\
\text { path from the source to the desti- } \\
\text { nation }\end{array}$ \\
\hline 71 & $\begin{array}{l}\text { IEEE } 802.11 \text { MAC proto- } \\
\text { col for Multi-hop Reserva- } \\
\text { tion [67] }\end{array}$ & 2011 & No & 2 & No & Yes & Large & Medium & Large & $\begin{array}{l}\text { Is designed for multi-hop reserva- } \\
\text { tion }\end{array}$ \\
\hline 72 & SAUCeR [90] & 2011 & Yes & 2 & Yes & No & Small & Small & Small & $\begin{array}{l}\text { Involves additional cooperative re- } \\
\text { transmissions and distributed relay } \\
\text { selection protocols }\end{array}$ \\
\hline
\end{tabular}

TABLE III: Comparison of QoS mechanisms used by MAC protocols

(BD - Backoff Differentiation, IFSD - IFS Differentiation, FM - Frame Manipulation, FA - Frame Aggregation, STR

- Stream Reservation, SLR - Slot Reservation, J - Jamming, CP/CFP - Alternating CP/CFP)

\begin{tabular}{|c|c|c|c|c|c|c|c|c|c|c|c|}
\hline & Protocol & BD & IFSD & FM & FA & STR & SLR & $\mathbf{J}$ & CP/CFP & Other & Year \\
\hline 1 & Black Burst [44] [45] & & $\bar{v}$ & & & & & $\boldsymbol{V}$ & & & 1996 \\
\hline 2 & MACA/PR [61] & & & & & $\boldsymbol{V}$ & & & & & 1997 \\
\hline 3 & EY-NPMA [91] & $\boldsymbol{V}$ & & $\boldsymbol{V}$ & & & & $\boldsymbol{V}$ & & & 1998 \\
\hline 4 & Priority scheme for DCF [23] & $\boldsymbol{V}$ & $\boldsymbol{V}$ & & & & & & & & 1999 \\
\hline 5 & RT-MAC [25] & $\boldsymbol{v}$ & & $\boldsymbol{V}$ & & & & & & & 1999 \\
\hline 6 & M-DCF [19] & $\boldsymbol{V}$ & & & & & & & & & 2000 \\
\hline 7 & SRMA/PA [68] & $\boldsymbol{V}$ & & $\boldsymbol{V}$ & & & $\boldsymbol{\sigma}$ & & & & 2000 \\
\hline 8 & $\begin{array}{l}\text { Extended DCF with Service Differentiation } \\
{[20]}\end{array}$ & $\boldsymbol{v}$ & & & & & & & & & 2001 \\
\hline 9 & Seedex [75] & & & & & & $\bar{v}$ & & & & 2001 \\
\hline 10 & DBASE [76] & & & & & & & & $\bar{v}$ & & 2001 \\
\hline 11 & PUMA [43] & & $\bar{V}$ & $\boldsymbol{V}$ & $\boldsymbol{V}$ & & & $\boldsymbol{V}$ & & & 2002 \\
\hline 12 & ES-DCF [41] & & $\bar{V}$ & $\boldsymbol{V}$ & & & & $\boldsymbol{V}$ & & & 2002 \\
\hline 13 & DB-DCF [41] & & $\checkmark$ & $V$ & & & & $V$ & & & 2002 \\
\hline 14 & DDRR [42] & & $\bar{V}$ & & & & & & & & 2002 \\
\hline 15 & DPS [48] & $\boldsymbol{V}$ & $\bar{\sigma}$ & $\boldsymbol{V}$ & & & & & & & 2002 \\
\hline 16 & D-PRMA [69] & & & & & & $\bar{\sigma}$ & & & & 2002 \\
\hline 17 & PBC [50] & $V$ & & & & & & $\boldsymbol{V}$ & & & 2003 \\
\hline 18 & AA [82] & & & & & & & & & $\boldsymbol{V}$ & 2003 \\
\hline 19 & MALT [81] & & & & & & & & & $\bar{v}$ & 2003 \\
\hline 20 & DDS [80] & & & & & & & & & $\boldsymbol{V}$ & 2003 \\
\hline 21 & WRT-Ring [87] & & & & & & & & & $\checkmark$ & 2003 \\
\hline 22 & CSMA/IC [53] & & & & & & & $\boldsymbol{V}$ & & & 2003 \\
\hline 23 & IFS Based Service Differentiation [36] & $\boldsymbol{V}$ & $\sqrt{ }$ & & & & & & & & 2003 \\
\hline 24 & MMACA/PR [62] & $\boldsymbol{V}$ & & & & $\boldsymbol{V}$ & & & & & 2003 \\
\hline 25 & NICER [79] & & & & & $\boldsymbol{V}$ & & & $\boldsymbol{V}$ & & 2003 \\
\hline 26 & P-MAC [51] & & & & & & & $\boldsymbol{V}$ & & & 2004 \\
\hline 27 & RACSS [38] & & $\bar{\sigma}$ & $\boldsymbol{V}$ & & & & & & & 2004 \\
\hline 28 & R-CSMA/CA [47] & & $\bar{\sigma}$ & & & & $\bar{V}$ & & $\bar{V}$ & & 2004 \\
\hline 29 & Local data-control schemes [34] & $\boldsymbol{V}$ & $\boldsymbol{V}$ & & & & & & & & 2004 \\
\hline 30 & D-LSMA [64] & & & & & $\boldsymbol{V}$ & & & & & 2004 \\
\hline 31 & QPART [32] & $\boldsymbol{V}$ & & & & & & & & & 2004 \\
\hline 32 & WTRP [88] & & & & & & & & & $\bar{v}$ & 2004 \\
\hline 33 & MPC-MAC [77] & & $\bar{V}$ & & & & & & $\boldsymbol{V}$ & & 2004 \\
\hline
\end{tabular}




\begin{tabular}{|c|c|c|c|c|c|c|c|c|c|c|c|}
\hline 34 & Frame switching based on PCF [78] & & $\checkmark$ & & & & & & $\checkmark$ & & 2004 \\
\hline 35 & DFIC [26] & & & & & & & $\boldsymbol{v}$ & & & 2004 \\
\hline 36 & CFIC [26] & $\boldsymbol{V}$ & & & & & & & & & 2004 \\
\hline 37 & EDCA [15] & $\boldsymbol{V}$ & $\boldsymbol{V}$ & & $\boldsymbol{v}$ & & & & & & 2005 \\
\hline 38 & DRCE [21] & $\boldsymbol{V}$ & $\checkmark$ & & & & & & & & 2005 \\
\hline 39 & DFS [27] & $\boldsymbol{V}$ & & & & & & & & & 2005 \\
\hline 40 & ETXOP [37] & $\boldsymbol{V}$ & $\checkmark$ & & $\boldsymbol{V}$ & & & & & & 2005 \\
\hline 41 & PGDMA [46] & & $\boldsymbol{V}$ & & & & & $\boldsymbol{V}$ & & & 2006 \\
\hline 42 & MAC-FM [65] & $\boldsymbol{V}$ & & & & $\checkmark$ & & & & & 2006 \\
\hline 43 & $\begin{array}{l}\text { New MAC Protocol with Pseudo-TDMA } \\
\text { Behavior [66] }\end{array}$ & $\boldsymbol{v}$ & 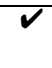 & & & $\boldsymbol{V}$ & & & & & 2006 \\
\hline 44 & EDCA/RR [58] & $\boldsymbol{V}$ & $\checkmark$ & & $\boldsymbol{V}$ & $\boldsymbol{V}$ & & & & & 2006 \\
\hline 45 & MAC-FM(E) [65] & $\boldsymbol{V}$ & & & & $\checkmark$ & & & $\checkmark$ & & 2006 \\
\hline 46 & QMA [54] & & & & & & & $\boldsymbol{V}$ & & & 2007 \\
\hline 47 & MMMP [60] & $\boldsymbol{V}$ & & $\checkmark$ & & $\checkmark$ & & & & & 2007 \\
\hline 48 & ReAllocative Priority [59] & $\boldsymbol{V}$ & $\boldsymbol{V}$ & $\boldsymbol{V}$ & $\boldsymbol{V}$ & & & & & & 2007 \\
\hline 49 & MPPC [63] & & & & & $\checkmark$ & & & & & 2007 \\
\hline 50 & C-MAC [35] & $\boldsymbol{V}$ & & & & $\boldsymbol{v}$ & & & & & 2007 \\
\hline 51 & DS-EDCA [29] & $\boldsymbol{V}$ & & & & & & & & & 2007 \\
\hline 52 & DSRP [70] & & & $\boldsymbol{V}$ & & & $\checkmark$ & & & & 2007 \\
\hline 53 & QoS-Croma [71] & & & & & & $\boldsymbol{V}$ & & & & 2007 \\
\hline 54 & Adaptive-TXOP (A-TXOP) [59] & $\boldsymbol{V}$ & $\checkmark$ & $\checkmark$ & $\boldsymbol{V}$ & & & & & & 2007 \\
\hline 55 & Clustering-based Multichannel MAC [83] & $\boldsymbol{V}$ & & & & & $\boldsymbol{V}$ & & & $\boldsymbol{V}$ & 2007 \\
\hline 56 & DS-MAC [39] & $\boldsymbol{V}$ & $\checkmark$ & & & & & & & $\boldsymbol{V}$ & 2008 \\
\hline 57 & PQAMP [30] & $\boldsymbol{V}$ & & $\boldsymbol{V}$ & & & & & & & 2008 \\
\hline 58 & MCTRP [85] & & & & & & & & & $\boldsymbol{V}$ & 2008 \\
\hline 59 & MCCA [93] & & & & $\boldsymbol{V}$ & $\boldsymbol{V}$ & & & & & 2008 \\
\hline 60 & HT-CDMA [84] & & & & & & & & & $\boldsymbol{v}$ & 2008 \\
\hline 61 & Busy-tone-based MAC [55] & 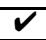 & $V$ & & & & & $\boldsymbol{V}$ & & & 2008 \\
\hline 62 & SPQAMP [30] & $\boldsymbol{V}$ & & & & & & & & & 2008 \\
\hline 63 & AQMP [31] & $\boldsymbol{V}$ & & & & & & & & & 2009 \\
\hline 64 & BusySiMOn [22] & $\boldsymbol{V}$ & $\boldsymbol{V}$ & & $\boldsymbol{v}$ & & & $\boldsymbol{V}$ & & & 2010 \\
\hline 65 & QCSCMA [86] & & & & & & & & & $\boldsymbol{V}$ & 2010 \\
\hline 66 & AMP [40] & $\boldsymbol{V}$ & $\checkmark$ & & & & & & & & 2010 \\
\hline 67 & DTSR [73] & & & & & & $\boldsymbol{V}$ & & & & 2010 \\
\hline 68 & T-MAC [74] & & & & & & $\boldsymbol{V}$ & & & & 2010 \\
\hline 69 & $\begin{array}{l}\text { Multi-channel MAC with service differenti- } \\
\text { ation [89] }\end{array}$ & $\boldsymbol{V}$ & & & & & & & & $\boldsymbol{V}$ & 2011 \\
\hline 70 & FQSA-MAC [33] & $\boldsymbol{V}$ & & & & & & & & & 2011 \\
\hline 71 & $\begin{array}{l}\text { IEEE 802.11 MAC protocol for Multi-hop } \\
\text { Reservation [67] }\end{array}$ & & & & & $\checkmark$ & & & & & 2011 \\
\hline 72 & SAUCeR [90] & & & & & & & & & $\checkmark$ & 2011 \\
\hline
\end{tabular}




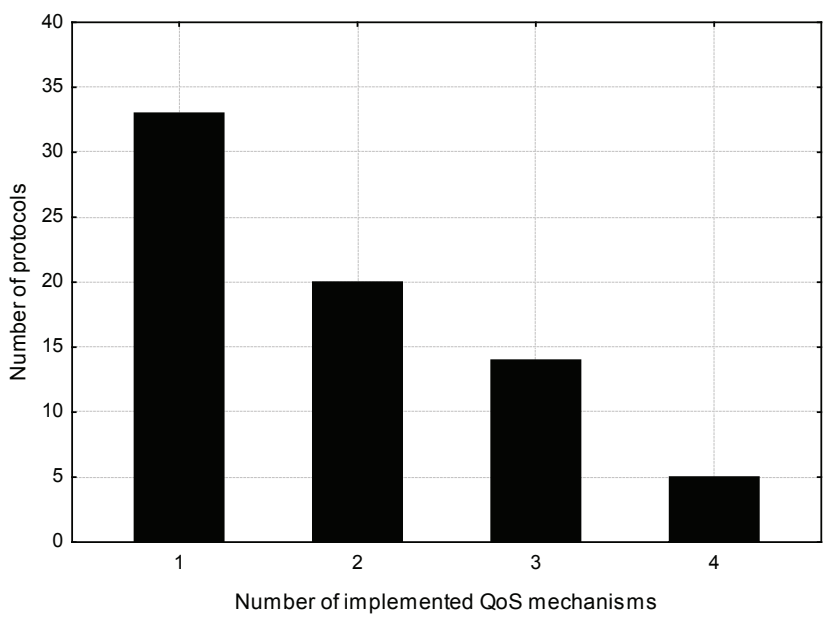

Fig. 19. Number of protocols versus number of implemented QoS mechanisms

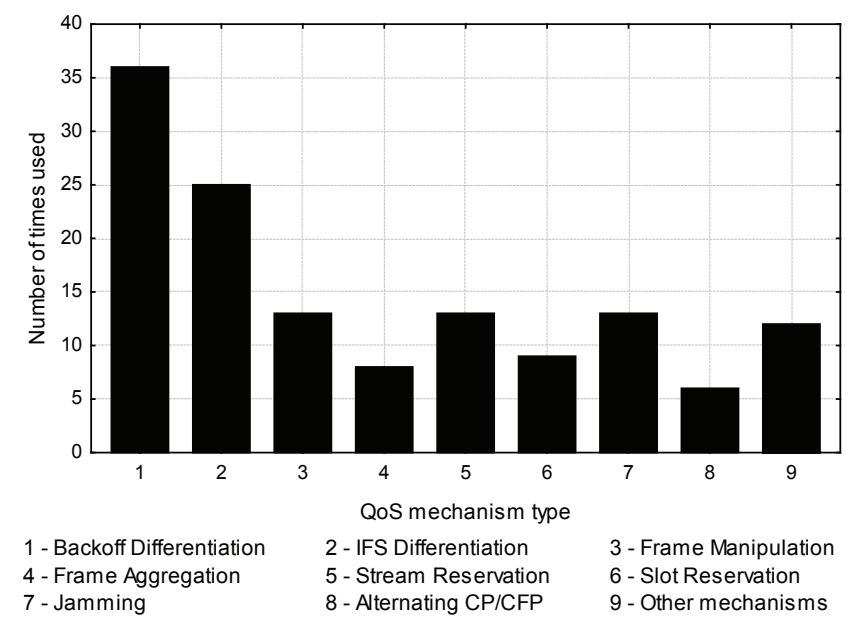

Fig. 20. Number of times used versus QoS mechanism type

Fig. 20 illustrates the number of times each of the QoS mechanisms was implemented by the protocols described in this survey. The backoff differentiation appears to be the most popular QoS mechanism. It was applied in more than 35 protocols. The second, only slightly less popular, is IFS differentiation. Different QoS mechanisms are not so commonly used. However, frame manipulation, stream reservation, jammingbased reservation are slightly more frequently used than frame aggregation, slot reservation, and alternating CP/CFP.

The total number of QoS-aware protocols published over the years is shown in Fig. 21. At the beginning, only one protocol was proposed each year. Then, the number of protocols grew exponentially until 2004. The largest number of new protocols was proposed in 2003, 2004, and 2007. Surprisingly, only one new protocol was published in 2009.

The quantitative usage of each QoS mechanism through the years is presented in Fig. 22. At first, only a small number of protocols implementing various QoS mechanisms were proposed. Six QoS mechanisms (out of the major eight described in this survey) were invented until 2000. The remaining two mechanisms were proposed in the following two years (2001-2002). Jamming and IFS differentiation were the

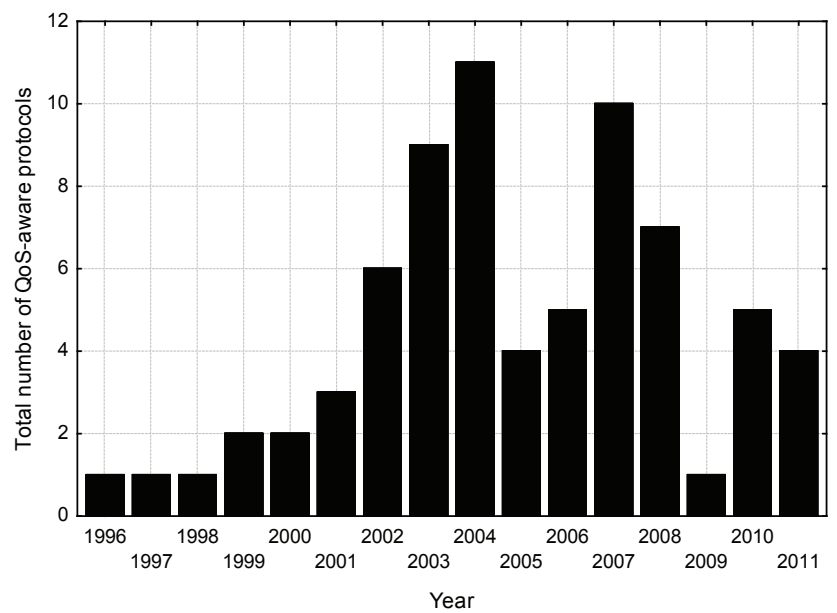

Fig. 21. Total numbers of QoS-aware protocols published over the years

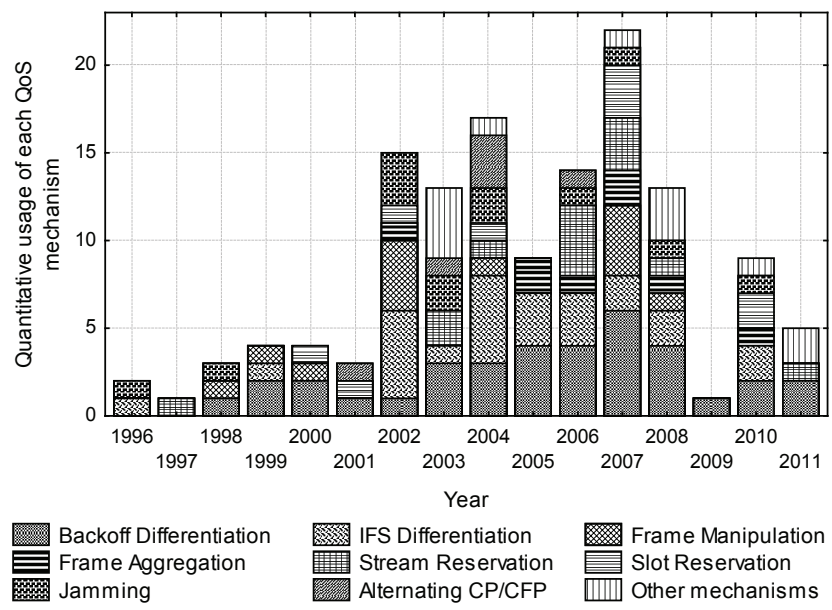

Fig. 22. Quantitative usage of each QoS mechanism through the years

first QoS mechanisms which were proposed in the literature while backoff differentiation has been consistently used since 1998. The largest number of new QoS-aware protocols as well as the variety of QoS mechanisms used occurred between 2002 and 2008. During that period, a large number of complex protocols was proposed (Table III). For several years, backoff and IFS differentiation have remained the most popular. However, various reservation mechanisms (e.g., stream or slot reservations) have also been constantly used. Unexpectedly, only one QoS mechanism based on backoff differentiation was employed in 2009.

\section{Open Issues and Future Research Directions}

Based on the presented survey three general comments related to the future research directions concerning QoS mechanisms can be derived. First of all, mechanisms which alternate contention and contention-free periods seem to be a promising solution for ad-hoc networks. In particular, contentionbased periods should be used for bursty traffic, reservationbased periods for real-time traffic, and broadcast periods for the dissemination of important information (e.g., reservation status). An alternative to such mechanisms could be a cross- 
layer approach. In particular, because as this survey has shown, it is impossible to provide strict QoS relying only on the MAC layer. A cross-layer solution is more complex, but may be unavoidable due to the nature of ad-hoc networks. An ideal cross-layer protocol should integrate QoS solutions from the physical, data link, and network layers as well as be able to extract information from upper layers (e.g., on traffic periodicity). The drawback of a cross-layer approach is that such solutions are difficult to implement and verify because of their complex architectures. Finally, most QoS MAC mechanisms proposed in the literature and described in this survey have been evaluated only through simulations. Such experiments are often based on unrealistic assumptions, e.g., ideal channel conditions. Hardware implementations and tests in real world deployments would give more insight on mechanism performance and lead to new mechanism proposals. Unfortunately, as mentioned in Section V-B, only the EDCA protocol has been implemented in commercial products.

In the following subsections two broad categories of open issues are described: specific challenges for future QoS mechanisms and general challenges for current and future ad-hoc networks.

\section{A. Specific Challenges for Future QoS Mechanisms}

The presented survey revealed several research gaps related to QoS mechanisms. First of all, it should be measured how the various existing QoS mechanisms influence each other. In the literature only several combinations were considered (e.g., the most commonly used combination of IFS and backoff differentiation) but it is possible that other combinations (e.g., a combination of mechanisms which form a protocol similar to Hybrid Coordination Function Controlled Channel Access [15], but designed for ad-hoc networks) would bring better results, which are currently difficult to predict.

Additionally, each mechanism (or combination of mechanisms) involves trade-offs between certain metrics (e.g., larger signaling overhead may lead to better delivery guarantees but increase latency). Therefore, trade-offs present in different mechanisms should be identified in order to find ways of minimizing them.

There may exist scenarios in which certain trade-offs are acceptable and may not necessarily negatively impact QoS (e.g., banking transactions require mobility support and strict delivery guarantees but not necessarily high transmission rates). Obviously, there may also exist scenarios in which certain trade-offs are unacceptable because they would impact the QoS provisioning. Therefore, if would be ideal if future QoS mechanisms could predict which trade-offs can be accepted and which should be avoided in the current state of their operation. They should also adjust their behavior accordingly to the recognized scenarios.

The shortcomings of the current QoS mechanisms should also be investigated in greater detail. Therefore, as proposed in [98], instead of the commonly applied analysis of high-level protocol performance (e.g., throughput and delay) low-level analysis is needed (i.e., each mechanism should be analyzed separately). Then, the mechanisms should be redesigned to eliminate (or at least minimize) their flaws and improve their performance.

Finally, novel QoS mechanisms should be proposed for currently available standards. They should be a response to the shortcomings of current QoS mechanisms, because it can be expected that not all existing flaws can be easily eliminated. This is because they are too strongly related to non-QoS characteristics of ad-hoc networks (e.g., wireless equipment constraints).

\section{B. General Challenges for Current and Future Ad-Hoc Net- works}

Based on the presented survey of QoS mechanisms for ad-hoc networks a number of general open issues can also be identified. First of all, several existing and well-known challenges of ad-hoc networks have not been adequately solved from the perspective of QoS. The hidden and exposed station problems are good examples of such challenges. Studies have shown problems with correct EDCA-based traffic differentiation in the presence of such stations [99] [100] [101] [102]. Energy saving MAC protocols can also be improved to be able to determine when stations can go into sleep mode without lowering the overall QoS provisioning of the network. Additionally, implementing QoS mechanisms in transmission power control MAC protocols is an interesting open issue. Furthermore, taking into account station mobility and assuring seamless handover would increase the QoS level in ad-hoc networks.

Furthermore, there are many other research directions which can be expected. In the near future, mesh networking will become a common form of wireless networking, especially with the recent release of IEEE 802.11s. With the increase of mesh deployments also the need for QoS support will grow. One of the main problems is the relaying of traffic of different priorities and with different QoS requirements. To this end, MAC protocols for mesh networks will certainly require to either cooperate with the network layer or adapt MAC-address based routing. Some work has already been done with respect to providing QoS in wireless mesh networks, especially related to MCCA (Section V-C), although more research can be expected. In particular, extensions to MCCA will surely be required, e.g., those defining the reservation parameters which are not specified in the standard.

Future research areas are also related to new radio and networking technologies. Among the former are cognitive and software defined radios. Additionally, future mobile stations will support better equipment, e.g., multiple transceivers, directional antennas, and a global positioning module. QoS MAC protocols will need to exploit these new features.

Among the novel networking technologies which are currently being developed, future ad-hoc networks will most likely embrace such concepts as virtualization and selfmanagement. QoS MAC protocols could also be equipped with these traits in order to improve their performance as well as lower the overall deployment and operating costs.

The application of new radio and networking technologies in ad-hoc networks will most likely be facilitated by open and modular programming architectures, such as SoftMAC [103] 
and FLAVIA [104]. They will allow everyone (from operators to end users), to individually program selected resource control functionalities. Modular architectures will greatly help in QoS provisioning by allowing the use of designs customized to the network conditions and user needs. However, additional steps need to be carried out so that intentional or unintentional misconfiguration does not decrease the level of QoS [105] [106].

Finally, other potential areas of future work include: accurate MAC-layer monitoring (for admission control), MAClayer support for multicast traffic, theoretical models of multihop networks, and dynamic QoS provisioning based on current network conditions.

\section{CONCLusions}

This paper has presented a broad overview of research related to QoS provisioning at the MAC layer in ad-hoc networks. Instead of describing each protocol individually we have decided to propose a novel taxonomic classification of QoS mechanisms, then outline the mechanisms one by one, and finally describe their protocol-specific implementations. This approach not only makes the topic easier to understand, but it also shows the various ways in which each of the mechanisms can be applied. Additionally, this approach is complementary to the design and implementation of future modular architectures.

The large number of mechanisms and protocols described in this survey reflect the importance of QoS-related research. This area of study is mostly challenging in the context of adhoc networks because of their frequently changing network topology, unstable channel conditions, station mobility, limited battery and computational power, etc. All these issues have been identified and commented in this survey.

For the reader's convenience, a comparison of protocols has been provided with the indication of important features and supported mechanisms. This was done in order to help the reader more broadly understand how the presented MAC protocols differ from each other. Furthermore, we have given short descriptions of current and evolving standards related to QoS provisioning at the MAC layer in ad-hoc networks and devoted a separate section to the most probable future research directions.

An important finding from the conducted research is that even despite broad research done in the area of QoS provisioning currently there is still no ideal QoS-aware solution which would meet all the expectations of users and network designers. There are groups of protocols which exhibit really good performance in certain, well defined scenarios, however, none of them is broad enough to resolve all of the problems appearing in ad-hoc networks.

\section{ACKNOWLEDGMENT}

This work has been supported by the European Community's Seventh Framework Program (FP7-ICT-2009-5) under grant agreement n. 257263 (FLAVIA project).

\section{REFERENCES}

[1] M. Natkaniec, K. Kosek-Szott, S. Szott, J. Gozdecki, A. Głowacz, and S. Sargento, "Supporting QoS in integrated ad-hoc networks," Wireless Personal Communications, vol. 56, pp. 183-206, 2011.

[2] S. Van den Heuvel-Romaszko and C. Blondia, "A survey of MAC protocols for ad hoc networks and IEEE 802.11," Available on line: http://www.pats.ua.ac.be/publications/2004/Nissi.pdf, 2004.

[3] H. Zhai, J. Wang, X. Chen, and Y. Fang, "Medium access control in mobile ad hoc networks: challenges and solutions," Wireless Coтmunications and Mobile Computing, vol. 6, pp. 151-170, 2006.

[4] H. Menouar, F. Filali, and M. Lenardi, "A survey and qualitative analysis of MAC protocols for vehicular ad hoc networks," IEEE Wireless Commun., vol. 13, pp. 30-35, 2006.

[5] O. Bazan and M. Jaseemuddin, "A survey on MAC protocols for wireless ad-hoc networks with beamforming antennas," IEEE Commun. Surveys Tuts., vol. 14, 2012.

[6] D. Perkins and H. Hughes, "A survey on quality-of-service support for mobile ad hoc networks," Wireless Communications and Mobile Computing, vol. 2, pp. 503-513, 2002.

[7] I. Jawhar and J. Wu, "QoS support in TDMA-based mobile ad hoc networks," J. Computer Science and Technology, vol. 20, pp. 797-810, 2005.

[8] T. Reddy, I. Karthigeyan, B. Manoj, and C. Murthy, "Quality of service provisioning in ad hoc wireless networks: a survey of issues and solutions," Ad Hoc Networks, vol. 4, pp. 83-124, 2006.

[9] S. Kumar, V. Raghavan, and J. Deng, "Medium Access Control protocols for ad hoc wireless networks: A survey," Ad Hoc Networks, vol. 4, pp. 326-358, 2006.

[10] O. Tsigkas and F. Pavudou, "Providing QoS support at the distributed wireless MAC layer: a comprehensive study," IEEE Wireless Commun., vol. 15, pp. 22-31, 2008.

[11] A. Abbas and O. Kure, "Quality of Service in mobile ad hoc networks: a survey," International Journal of Ad Hoc and Ubiquitous Computing, vol. 6, pp. 75-98, 2010.

[12] M. Natkaniec, K. Kosek-Szott, and S. Szott, "QoS Support in Multihop Ad-hoc Networks," in Wireless Network Traffic and Quality of Service Support: Trends and Standards, T. Lagkas, P. Angelidis, and L. Georgiadis, Eds. IGI Global, 2010.

[13] S. Shenker, R. Braden, and D. Clark, "Integrated services in the internet architecture: an overview," IETF Request for Comments (RFC), vol. 1633, 1994.

[14] S. Blake, D. Black, M. Carlson, E. Davies, Z. Wang, and W. Weiss, "An architecture for differentiated services," IETF Request for Comments (RFC), vol. 2475, 1998.

[15] I. . W. Group et al., IEEE 802.11-2007: Wireless LAN Medium Access Control (MAC) and Physical Layer (PHY) Specifications, IEEE Std., 2007.

[16] M. Natkaniec and A. R. Pach, "Performance analysis of IEEE 802.11 networks in the presence of hidden stations," Proc. Personal Wireless Communications (PWC), pp. 157-168, 2000.

[17] Y. Li, S. Mao, and S. Panwar, "PSMAC: Polling Service-based Medium Access Control for Wireless Networks," in Modeling and Optimization in Mobile, Ad Hoc and Wireless Networks and Workshops, 2007. WiOpt 2007. 5th International Symposium on, 2007.

[18] Y. Li, S. Mao, S. Panwar, and S. Midkiff, "On the performance of distributed polling service-based medium access control," IEEE Trans. on Wireless Commun., vol. 7, pp. 4635 -4645, 2008.

[19] J. Xiaohui, L. Jiandong, and G. Feng, "M-DCF: a MAC protocol supporting QoS in ad hoc network," in Communication Technology Proc., 2000. WCC-ICCT 2000. International Conference on, 2000.

[20] A. Veres, A. Campbell, M. Barry, and L. Sun, "Supporting service differentiation in wireless packet networks using distributed control," IEEE J. Sel. Areas Commun., vol. 19, pp. 2081-2093, 2001.

[21] T. You, C. Yeh, and H. Hassanein, "DRCE: a high throughput QoS MAC protocol for wireless ad hoc networks," in Computers and Communications, 2005. ISCC 2005. Proc. 10th IEEE Symposium on, 2005.

[22] K. Kosek-Szott, M. Natkaniec, and A. R. Pach, "BusySiMOn-a new protocol for IEEE 802.11 EDCA-based ad-hoc networks with hidden nodes," in GLOBECOM 2010, 2010 IEEE Global Telecommunications Conference, 2010.

[23] D. Deng and R. Chang, "A priority scheme for IEEE 802.11 DCF access method," IEICE Trans. Commun. E Series B, vol. 82, pp. 96$102,1999$.

[24] N. Sarma, A. Singh, and S. Nandi, "A strict priority based QoS-aware MAC protocol for mobile ad hoc networks," Distributed Computing and Internet Technology, pp. 121-132, 2009. 
[25] R. Baldwin, N. Davis IV, and S. Midkiff, "A real-time medium access control protocol for ad hoc wireless local area networks," $A C M$ SIGMOBILE Mobile Computing and Communications Review, vol. 3, pp. 20-27, 1999.

[26] T. You, H. Hassanein, and C. Yeh, "Controllable fair QoS-based MAC protocols for ad hoc wireless networks," in Proc. IEEE International Workshop on Mobile and Wireless Networking, 2004.

[27] N. Vaidya, A. Dugar, S. Gupta, and P. Bahl, "Distributed fair scheduling in a wireless LAN," IEEE Trans. Mobile Computing, vol. 4, pp. 616 $-629,2005$

[28] S. Golestani, "A self-clocked fair queueing scheme for broadband applications," in INFOCOM'94. Networking for Global Communications., 13th Proc. IEEE, 1994.

[29] J. Lee, W. Liao, and M. Chen, "A differentiated service model for enhanced distributed channel access (EDCA) of IEEE 802.11e WLANs," Mobile Networks and Applications, vol. 12, pp. 69-77, 2007.

[30] N. Sarma and S. Nandi, "A priority based QoS-aware MAC protocol (PQAMP) in mobile ad hoc networks," in Proc. 4th ACM symposium on QoS and security for wireless and mobile networks, 2008.

[31] R. Geng, Z. Li, and L. Song, "AQMP: An Adaptive QoS MAC Protocol Based on IEEE 802.11 in Ad Hoc Networks," in Proc. 5th International Conference on Wireless Communications, Networking and Mobile Computing - WiCom'09, 2009.

[32] Y. Yang and R. Kravets, "Distributed QoS guarantees for realtime traffic in ad hoc networks," in Sensor and Ad Hoc Communications and Networks, 2004. IEEE SECON 2004. 2004 First Annual IEEE Communications Society Conference on, 2004.

[33] D. Seth, S. Patnaik, and S. Pal, "A Faired Quality of Service Assured MAC Protocol for Mobile ADHOC Network and its Performance Evaluation," International Journal of Wireless and Mobile Networks, vol. 3, 2011.

[34] Y. Xiao and H. Li, "Local data control and admission control for QoS support in wireless ad hoc networks," IEEE Trans. Veh. Technol., vol. 53, pp. 1558-1572, 2004

[35] L. Wang, A. Chen, and D. Wei, "A cognitive MAC protocol for QoS provisioning in overlaying ad hoc networks," in Consumer Communications and Networking Conference, 2007. CCNC 2007. 4th IEEE, 2007.

[36] C. Chou, K. Shin, and S. Shankar, "Inter-frame space (ifs) based service differentiation for IEEE 802.11 wireless LANs," in Vehicular Technology Conference, 2003. VTC 2003-Fall. 2003 IEEE 58th, 2003.

[37] A. Ksentini, A. Gueroui, and M. Naimi, "A new IEEE 802.11 MAC protocol with admission control for sensitive multimedia applications," in Global Telecommunications Conference, 2005. GLOBECOM'05. IEEE, 2005.

[38] I. Cârdei and S. Kazi, "MAC layer QoS support for wireless networks of unmanned air vehicles," in System Sciences, 2004. Proc. 37th Annual Hawaii International Conference on, 2004.

[39] X. Yuan, S. Bagga, J. Shen, M. Balakrishnan, and D. Benhaddou, "DSMAC: Differential service medium access control design for wireless medical information systems," in Engineering in Medicine and Biology Society, 2008. EMBS 2008. 30th Annual International Conference of the IEEE, 2008.

[40] R. Geng, L. Guo, and X. Wang, "A new adaptive MAC protocol with QoS support based on IEEE 802.11 in ad hoc networks," Computers \& Electrical Engineering, 2010.

[41] A. Pal, A. Dogan, and F. Ozguner, "MAC layer protocols for realtime traffic in ad-hoc wireless networks," in Parallel Processing, 2002. Proceedings. International Conference on, 2002.

[42] W. Pattara-Aukom, S. Banerjee, and P. Krishnamurthy, "Starvation prevention and quality of service in wireless LANs," in Wireless Personal Multimedia Communications, 2002. The 5th International Symposium on, 2002.

[43] M. Natkaniec and A. R. Pach, "PUMA-a new channel access protocol for wireless LANs," in Wireless Personal Multimedia Communications, 2002. The 5th International Symposium on, 2002.

[44] J. Sobrinho and A. Krishnakumar, "Quality-of-service in ad hoc carrier sense multiple access wireless networks," IEEE J. Sel. Areas Commun., vol. 17, pp. 1353-1368, 1999.

[45] —, "Real-time traffic over the IEEE 802.11 medium access control layer," Bell Labs Technical Journal, vol. 1, pp. 172-187, 1996.

[46] F. Eshghi and V. Krishnamurthy, "Wlc15-6: A QoS-based MAC protocol for ad hoc WLANs," in Global Telecommunications Conference, 2006. GLOBECOM'O6. IEEE, 2006.

[47] I. Joe, "QoS-aware MAC with reservation for mobile ad-hoc networks," in Vehicular Technology Conference, 2004. VTC2004-Fall. 2004 IEEE 60th, 2004.
[48] V. Kanodia, C. Li, A. Sabharwal, B. Sadeghi, and E. Knightly, "Distributed priority scheduling and medium access in ad hoc networks," Wireless Networks, vol. 8, pp. 455-466, 2002.

[49] G. Bianchi and I. Tinnirello, "Analysis of priority mechanisms based on differentiated inter frame spacing in CSMA-CA," in Vehicular Technology Conference, 2003. VTC 2003-Fall. 2003 IEEE 58th, 2003.

[50] C. Yeh and T. You, "A QoS MAC protocol for differentiated service in mobile ad hoc networks," in Parallel Processing, 2003. Proceedings. 2003 International Conference on, 2003.

[51] J. Sheu, C. Liu, S. Wu, and Y. Tseng, "A priority MAC protocol to support real-time traffic in ad hoc networks," Wireless networks, vol. 10, pp. 61-69, 2004.

[52] K. Nakano and S. Olariu, "Randomized initialization protocols for ad hoc networks," IEEE Trans. Parallel Distrib. Syst., vol. 11, pp. 749759, 2000.

[53] T. You, C. Yeh, and H. Hassanein, "CSMAIC: A new class of collisionfree MAC protocols for ad hoc wireless networks," in Computers and Communication, 2003.(ISCC 2003). Proceedings. Eighth IEEE International Symposium on, 2003.

[54] D. Wang and K. Liu, "A novel QoS MAC protocol for multi-hop ad hoc networks," in Wireless Communications, Networking and Mobile Computing, 2007. WiCom 2007. International Conference on, 2007.

[55] P. Wang, H. Jiang, and W. Zhuang, "A new MAC scheme supporting voice/data traffic in wireless ad hoc networks," IEEE Trans. Mobile Computing, vol. 7, pp. 1491-1503, 2008.

[56] Z. Haas and J. Deng, "Dual busy tone multiple access (DBTMA)a multiple access control scheme for ad hoc networks," IEEE Trans. Commun., vol. 50, pp. 975-985, 2002.

[57] J. Garcia-Luna-Aceves and C. Fullmer, "Floor acquisition multiple access (FAMA) in single-channel wireless networks," Mobile Networks and Applications, vol. 4, pp. 157-174, 1999.

[58] A. Hamidian and U. Körner, "An enhancement to the IEEE 802.11e EDCA providing QoS guarantees," Telecommunication Systems, vol. 31, pp. 195-212, 2006.

[59] T. Bheemarjuna Reddy, J. John, and C. Murthy, "Providing MAC QoS for multimedia traffic in $802.11 \mathrm{e}$ based multi-hop ad hoc wireless networks," Computer Networks, vol. 51, pp. 153-176, 2007.

[60] M. Sarkar, S. Gurajala, and S. Kumar, "A MAC protocol to support QoS for multimedia traffic transmission over ad hoc networks," in Proc. 2007 international conference on Wireless communications and mobile computing, 2007.

[61] C. Lin and M. Gerla, "Asynchronous multimedia multihop wireless networks," in INFOCOM '97. Sixteenth Annual Joint Conference of the IEEE Computer and Communications Societies. Proceedings IEEE, 1997.

[62] Z. Ying, A. Ananda, and L. Jacob, "A QoS enabled MAC protocol for multi-hop ad hoc wireless networks," in Performance, Computing, and Communications Conference, 2003. Conference Proc. 2003 IEEE International, april 2003, pp. 149 - 156.

[63] M. Sarkar and S. Borra, "A QoS and power aware MAC layer protocol for wireless ad hoc networks," in World Congress on Engineering and Computer Science 2008, WCECS'08. Advances in Electrical and Electronics Engineering-IAENG Special Edition of the, 2008.

[64] Z. Wu and D. Raychaudhuri, "D-LSMA: Distributed link scheduling multiple access protocol for QoS in ad-hoc networks," in Global Telecommunications Conference, 2004. GLOBECOM'04. IEEE, 2004.

[65] Q. Jigang, L. Huijie, and L. Xiaokang, "The MAC mechanism with QoS provision based on stream-maintenance in ad hoc networks," in ITS Telecommunications Proceedings, 2006 6th International Conference on, 2006.

[66] G. Paschos, I. Papapanagiotou, S. Kotsopoulos, and G. Karagiannidis, "A new MAC protocol with pseudo-tdma behavior for supporting quality of service in 802.11 wireless LANs," EURASIP Journal on Wireless Communications and Networking, vol. 2006, p. 8, 2006.

[67] Y. Cho, S. Yoon, and Y. Ko, "Modifying the IEEE 802.11 MAC Protocol for Multi-hop Reservation in MIMC Tactical Ad Hoc Networks," in Advanced Information Networking and Applications (WAINA), 2011 IEEE Workshops of International Conference on, 2011.

[68] C. W. Ahn, C. G. Kang, and Y. Z. Cho, "Soft reservation multiple access with priority assignment (SRMA/PA): a novel MAC protocol for QoS-guaranteed integrated services in mobile ad-hoc networks," in Vehicular Technology Conference, 2000. IEEE VTS-Fall VTC 2000. 52nd, 2000.

[69] S. Jiang, J. Rao, D. He, X. Ling, and C. Ko, "A simple distributed PRMA for MANETs,” IEEE Trans. Veh. Technol., vol. 51, pp. 293 $305,2002$.

[70] Y. Zhang, X. Sun, and J. Li, "A distributed synchronous reservation multiple access control protocol for mobile ad hoc networks," Frontiers 
of Electrical and Electronic Engineering in China, vol. 2, pp. 68-72, 2007.

[71] F. De Rango, A. Perrotta, and S. Marano, "QoS-CROMA: An ondemand time-slotted MAC protocol with QoS support for wireless ad hoc networks," in Wireless Communication Systems, 2007. ISWCS 2007. 4th International Symposium on, 2007.

[72] A. Silberschatz, P. Galvin, G. Gagne, and A. Silberschatz, Operating system concepts. Addison-Wesley, 1998.

[73] S. Kamruzzaman and M. Alam, "Dynamic TDMA slot reservation protocol for cognitive radio ad hoc networks," in Computer and Information Technology (ICCIT), 2010 13th International Conference on, 2010.

[74] Y. Wang, P. Ren, and G. Wu, "A throughput-aimed MAC protocol with QoS provision for cognitive ad hoc networks," IEICE Trans. Commun. E, vol. 93, pp. 1426-1429, 2010.

[75] R. Rozovsky and P. Kumar, "Seedex: A MAC protocol for ad hoc networks," in Proc. 2nd ACM international symposium on Mobile ad hoc networking \& computing, 2001.

[76] S. Sheu and T. Sheu, "A bandwidth allocation/sharing/extension protocol for multimedia over IEEE 802.11 ad hoc wireless LANs," IEEE J. Sel. Areas Commun., vol. 19, pp. 2065-2080, 2001.

[77] T. You, H. Hassanein, and H. Mouftah, "Infrastructure-based MAC in wireless mobile ad-hoc networks," in Local Computer Networks, 2002. Proceedings. LCN 2002. 27th Annual IEEE Conference on, 2002.

[78] S. Sivavakeesar and G. Pavlou, "Quality of service aware MAC based on IEEE 802.11 for multihop ad-hoc networks," in Wireless Communications and Networking Conference, 2004. WCNC. 2004 IEEE, 2004.

[79] A. Chen, L. Wang, C. Wang, and D. Wei, "NICER - a distributed wireless MAC protocol for mobile ad hoc networks," in Vehicular Technology Conference, 2003. VTC 2003-Fall. 2003 IEEE 58th, 2003.

[80] C. Yeh, "QoS differentiation mechanisms for heterogeneous wireless networks and the next-generation internet," in Computers and Communication, 2003.(ISCC 2003). Proceedings. Eighth IEEE International Symposium on, 2003.

[81] C. Yeh and T. You, "A power-controlled multiple access scheme for differentiated service and energy efficiency in mobile ad hoc networks and wireless LANs," in Personal, Indoor and Mobile Radio Communications, 2003. PIMRC 2003. 14th IEEE Proceedings on, 2003.

[82] C. Yeh, "The advance access mechanism for differentiated service, power control, and radio efficiency in ad hoc MAC protocols," in Vehicular Technology Conference, 2003. VTC 2003-Fall. 2003 IEEE 58th, 2003.

[83] H. Su and X. Zhang, "Clustering-based multichannel MAC protocols for QoS provisionings over vehicular ad hoc networks," IEEE Trans. Veh. Technol., vol. 56, pp. 3309-3323, 2007.

[84] I. Liu, F. Takawira, and H. Xu, "A hybrid token-cdma MAC protocol for wireless ad hoc networks," IEEE Trans. Mobile Computing, vol. 7, pp. 557-569, 2008.

[85] Y. Bi, K. Liu, X. Shen, and H. Zhao, "A multi-channel token ring protocol for inter-vehicle communications," in Global Telecommunications Conference, 2008. IEEE GLOBECOM 2008. IEEE, 2008.

[86] J. Zheng and M. Ma, "QoS-aware cooperative medium access control for MIMO ad-hoc networks," IEEE Commun. Lett., vol. 14, pp. 48-50, 2010.

[87] L. Donatiello and M. Furini, "Ad hoc networks: a protocol for supporting QoS applications," in Parallel and Distributed Processing Symposium, 2003. Proceedings. International, 2003.

[88] M. Ergen, D. Lee, R. Sengupta, and P. Varaiya, "WTRP-wireless token ring protocol," IEEE Trans. Veh. Technol., vol. 53, pp. 1863-1881, 2004.

[89] K. Shin, S. Yun, and D. Cho, "Multi-channel MAC protocol for QoS support in ad-hoc network," in Consumer Communications and Networking Conference (CCNC), 2011 IEEE, 2011.

[90] H. Tan, M. Chan, P. Kong, and C. Tham, "SAUCeR: a QoS-aware slotted-aloha based uwb MAC with cooperative retransmissions," Wireless Communications and Mobile Computing, vol. 11, pp. 410-425, 2011

[91] EN 300652 v1.2.1: Broadband Radio Access Networks (BRAN): HIgh PERformance Radio Access Network (HIPERLAN) Type I: Functional Specification, ETSI Std., 1998.

[92] A. Warrier and I. Rhee, "Rethinking wireless MAC architecture for quality of service support-design and implementation," 2007, published online.

[93] IEEE Standard for Information Technology-Telecommunications and information exchange between systems-Local and metropolitan area networks-Specific requirements Part 11: Wireless LAN Medium Access
Control (MAC) and Physical Layer (PHY) specifications Amendment 10: Mesh Networking, IEEE Std., 2011.

[94] G. Hiertz, Y. Zang, S. Max, T. Junge, E. Weiss, and B. Wolz, "IEEE 802.11s: WLAN mesh standardization and high performance extensions," Network, IEEE, vol. 22, pp. 12-19, 2008.

[95] IEEE Standard for Information technology-Telecommunications and information exchange between systems Local and metropolitan area networks-Specific requirements Part 11: Wireless LAN Medium Access Control (MAC) and Physical Layer (PHY) Specifications Amendment 2: MAC Enhancements for Robust Audio Video Streaming, IEEE Std., 2012.

[96] IEEE Standard for Information technology-Telecommunications and information exchange between systems-Local and metropolitan area networks-Specific requirements Part 11: Wireless LAN Medium Access Control (MAC) and Physical Layer (PHY) Specifications Amendment 1: Prioritization of Management Frames, IEEE Std., 2012.

[97] M. Natkaniec and A. R. Pach, "An analysis of modified backoff mechanism in IEEE 802.11 networks," in First Polish-German Teletraffic Symposium (PGTS2000), 2000.

[98] G. Bianchi, I. Tinnirello, and L. Scalia, "Understanding 802.11e contention-based prioritization mechanisms and their coexistence with legacy 802.11 stations," IEEE Network, vol. 19, pp. 28-34, 2005.

[99] K. Kosek, M. Natkaniec, L. Vollero, and A. R. Pach, "Performance analysis of 802.11e networks with hidden nodes in a star topology," in Consumer Communications and Networking Conference, 2008. CCNC 2008. 5th IEEE, 2008.

[100] K. Kosek, M. Natkaniec, and L. Vollero, "Thorough analysis of ieee 802.11 EDCA in ring topology scenarios with hidden and exposed nodes," Computational Science and Its Applications-ICCSA 2009, pp. 636-648, 2009.

[101] K. Kosek, M. Natkaniec, and A. R. Pach, "Analysis of IEEE 802.11e line topology scenarios in the presence of hidden nodes," Ad-hoc, Mobile and Wireless Networks, pp. 380-390, 2008.

[102] K. Kosek, M. Natkaniec, and L. Vollero, "Thorough analysis of 802.11e star topology scenarios in the presence of hidden nodes," in Proc. 7th international IFIP-TC6 networking conference on AdHoc and sensor networks, wireless networks, next generation internet, 2008.

[103] M. Neufeld, J. Fifield, C. Doerr, A. Sheth, and D. Grunwald, "Softmacflexible wireless research platform," in Proc. HotNets-IV, 2005.

[104] "Flexible architecture for virtualizable future wireless internet access (flavia)," http://www.ict-flavia.eu/.

[105] S. Szott, M. Natkaniec, R. Canonico, and A. Pach, "Impact of contention window cheating on single-hop IEEE 802.11e MANETs," in Wireless Communications and Networking Conference, 2008. WCNC 2008. IEEE, 2008.

[106] S. Szott, "Assuring QoS in wireless mesh networks with misbehaving users," in INFOCOM Workshops 2009, IEEE, 2009.

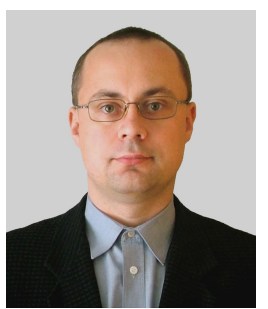

Marek Natkaniec Marek Natkaniec received the M.Sc. and Ph.D. degrees in telecommunications from the AGH University of Science and Technology, Krakow, Poland in 1997 and 2002, respectively. In 1997 he joined AGH University of Science and Technology as a researcher. Now, he works as an assistant professor at the Department of Telecommunications. His general research interests are in wireless networks. Particular topics include wireless LANs, protocol design, modeling and performance evaluation of communication networks, quality of service, and cooperation of networks. He is a reviewer for international journals and conferences. He has actively participated in several European projects (MOCOMTEL, PRO-ACCESS, DAIDALOS I, DAIDALOS II, CONTENT, CARMEN, MEDUSA, HECTOR, FLAVIA, PROACTIVE) as well as grants supported by the Ministry of Science and Higher Education. He is involved in standardization activities for ETSI. He serves as an expert and a consultant to telecom operators in the area of wireless networks. Marek Natkaniec has co-authored five books and over 100 research papers. He is a senior member of IEEE. 


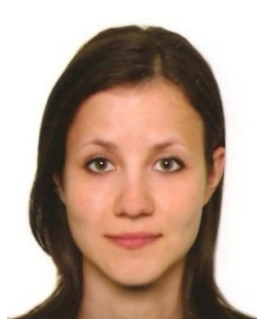

Katarzyna Kosek-Szott Katarzyna Kosek-Szott received her M.Sc. and Ph.D. degrees in telecommunications (both with honours) from the AGH University of Science and Technology, Krakow, Poland in 2006 and 2011, respectively. Currently she is working as an assistant professor at the Department of Telecommunications, AGH University of Science and Technology. Her general research interests are focused on wireless networking. The major topics include wireless LANs (especially ad-hoc networks) and quality of service provisioning within these networks. She is a reviewer for international journals and conferences. She has been involved in several European projects: DAIDALOS II, CONTENT, CARMEN, FLAVIA, PROACTIVE as well as grants supported by the Polish Ministry of Science and Higher Education. She is involved in standardization activities for ETSI. She has co-authored one book chapter and a number of research papers.

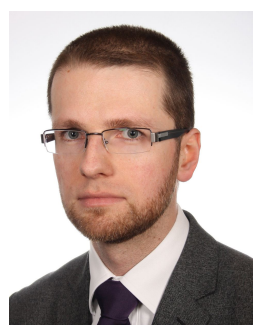

Szymon Szott Szymon Szott received his M.Sc. and $\mathrm{Ph} . \mathrm{D}$. degrees in telecommunications (both with honours) from the AGH University of Science and Technology, Krakow, Poland in 2006 and 2011, respectively. Currently he is working as an assistant professor at the Department of Telecommunications, AGH University. His professional interests are related to wireless networks (in particular: QoS provisioning and security of ad-hoc networks). He is a reviewer for international journals and conferences. He has been involved in several European projects (DAIDALOS II, CONTENT, CARMEN, MEDUSA, FLAVIA, PROACTIVE) as well as grants supported by the Ministry of Science and Higher Education and the National Science Centre. He serves as secretary for the ETSI Industry Specification Group "Autonomic network engineering for the self-managing Future Internet" (AFI). He is the co-author of over 30 research papers and one book chapter.

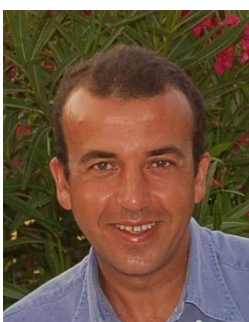

Giuseppe Bianchi Giuseppe Bianchi is Full Professor of Telecommunications at the School of Engineering of the University of Roma Tor Vergata since January 2007. He was formerly employed at Politecnico di Milano and University of Palermo. He spent 1992 as visitor researcher at the Washington University of St. Louis, Missouri, USA, and 1997 as visitor researcher at the Columbia University of New York. His research activity (published on about 170 papers in peer-refereed international journals and conferences) spans several areas, current active topics being wireless networks, network security, network measurement and monitoring, privacy. G. Bianchi has been involved in coordination roles (general, technical or scientific coordinator) for three European STREP projects, one European IP project, and two national PRIN projects, and has participated as unit coordinator to many other projects. He's area editor of IEEE Transactions on Wireless Communication, editor for IEEE/ACM Transactions on Networking, and area editor for Elsevier Computer Communications. He has chaired more than 10 international IEEE/ACM conferences or workshops. 